\title{
A técnica do super-passo na resolução numérica de equações diferenciais parciais parabólicas
}

\author{
Aimberê Galdino \\ DISSERTAÇÃO APRESENTADA \\ $\mathrm{AO}$ \\ INSTITUTO DE MATEMÁTICA E ESTATÍSTICA \\ DA \\ UNIVERSIDADE DE SÃO PAULO \\ PARA \\ OBTENÇÃO DO TÍTULO DE MESTRE \\ EM \\ CIÊNCIAS \\ Área de Concentração: Matemática Aplicada \\ Orientador: Prof. Dr. Alexandre Megiorin Roma
}

Durante a elaboração deste trabalho o autor recebeu auxílio financeiro do $\mathrm{CNPq}$

São Paulo, Junho de 2006 


\title{
A Técnica do Super-Passo na Resolução Numérica de Equações Diferenciais Parciais Parabólicas
}

\author{
Este exemplar corresponde à redação \\ final da dissertação devidamente cor- \\ rigida e defendida por Aimberê Galdino \\ e aprovada pela Comissão Julgadora.
}

São Paulo, 2 junho de 2006.

Banca examinadora:

- Prof. Dr. Alexandre Megiorin Roma (Orientador) - IME-USP

- Prof. Dr. Clodoaldo Grotta Ragazzo - IME-USP

- Prof. Dr. José Jaime da Cruz - POLI-USP 
Dedico este trabalho aos meus pais Gerson e Alice, ao tio Alexandre e a Lilian. 


\section{AGRADECIMENTOS}

À meu orientador Prof. Dr. Alexandre Roma, pela paciência e dedicação em me orientar durantes esses anos de pesquisa. Ao Prof. Dr. Vasilios Alexiades por estar, mesmo a distância, ajudando no desenvolvimento deste trabalho.

Aos meus pais, tio Alexandre, aos meus amigos de Santana de Parnaíba e a Lilian que sempre me apoiaram, incentivaram e ajudaram na conclusão deste trabalho.

Agradeço a todos os colegas de pós-graduação que me ajudaram na conclusão do mestrado.

Aos professores que compuseram a banca examinadora, pela leitura cuidadosa deste trabalho e sugestões ou críticas decorrentes da mesma.

Agradeço ao IME-USP, em especial a Comissão de Pós-Graduação pela oportunidade de realizar meus estudos nesta instituição.

Ao CNPq pela bolsa de pesquisa durante o mestrado. 


\section{Resumo}

A Técnica do Super-Passo pode melhorar significantemente a performance do Método de Euler Explícito, reduzindo a restrição existente ao passo no tempo. A técnica é descrita para a equação do calor linear. É mostrada a simplicidade de sua implementação para o caso do Método de Euler Explícito. A perfomance da Técnica do Super-Passo é comparada aos Métodos de Euler Explícito e Implícito, e Crank-Nicolson. Os resultados obtidos sugerem que o Super-Passo pode melhorar a eficiência do Método de Euler Explícito em aproximadamente uma ordem de grandeza reduzindo o tempo de processamento, enquanto que o erro produzido pela Técnica do Super-Passo é comparável ao produzido pelo Método de Euler Implícito. 


\section{Abstract}

The Super-Time-Stepping Technique can significantly increase the performance of the Explícit Euler Method, reducing the existing tie step restriction. The Techinique is described for a linear heat equation. The simplicity of this implementation for the case of the Explícit Euler Method is shown. The perfomance of the Technique of the Super-Time-Stepping is compared to Explicit and Implicit Euler, and Crank-Nicolson Methods. The obtained results suggest that the Technique of the Super-Time-Stepping potentially increases the efficiency of the Explicit Euler Method approximately an order of magnitude regarding the processing time, while the error produced is comparable to that produced by the Implicit Euler Method. 


\section{Sumário}

Lista de Figuras

Lista de Tabelas $\quad$ v

$\begin{array}{ll}\text { Introdução } & 1\end{array}$

1 Modelagem Matemática da Condução do Calor 4

1.1 Equações Diferenciais Parciais . . . . . . . . . . . . . . . . . 4

1.2 Classificação . . . . . . . . . . . . . . . . . . . 5

1.3 Modelagem Matemática da Propagação de Calor . . . . . . . . 6

2 Métodos Numéricos Clássicos $\quad 11$

2.1 Discretização: Diferenças Finitas . . . . . . . . . . . . . . . 11

2.2 Método de Euler Explícito . . . . . . . . . . . . . . . . 18

2.3 Método de Euler Implícito . . . . . . . . . . . . . . . . . 23

2.4 Método de Crank-Nicolson . . . . . . . . . . . . . . . . 27

3 A Técnica do Super-Passo 32

3.1 Condição de Estabilidade . . . . . . . . . . . . . . . . . 35

3.2 Consistência . . . . . . . . . . . . . . . . . . . 40

3.3 Erro Cometido na Aproximação pela Técnica do Super-Passo . 40 
4 Resultados Numéricos $\quad 44$

4.1 Análise Numérica de Convergência . . . . . . . . . . . . . . . 44

4.1.1 Método de Euler Explícito . . . . . . . . . . . . . 47

4.1 .2 Método de Euler Implícito . . . . . . . . . . . . . 47

4.1.3 Método de Crank-Nicolson . . . . . . . . . . . . . . . . 48

4.1.4 Técnica do Super-Passo . . . . . . . . . . . . . . . . 49

4.2 Comparação de Performance . . . . . . . . . . . . . . . . . . 51

5 Conclusão $\quad 55$

Apêndice: Polinômios de Chebyshev $\quad 57$

$\begin{array}{ll}\text { Referências Bibliográficas } & 63\end{array}$ 


\section{Lista de Figuras}

1.1 Propagação do calor em uma barra. . . . . . . . . . . . 7

2.1 Discretização do domínio $\Omega$. . . . . . . . . . . . . . 12

2.2 Molécula computacional do Método de Euler Explícito. . . . . 19

2.3 Molécula computacional do Método de Euler Implícito. . . . . 24

2.4 Molécula computacional do Método de Crank-Nicolson. . . . . 29

5.1 Polinômios de Chebyshev. . . . . . . . . . . . . . . 61 


\section{Lista de Tabelas}

3.1 Aproximação numérica para o limite (3.15). . . . . . . . . . . 39

4.1 Resultados numéricos obtidos pelo Método de Euler Explícito na aproximação da solução do problema (4.6). . . . . . . . . . 47

4.2 Resultados numéricos obtidos pelo Método de Euler Implícito na aproximação da solução do problema (4.6) . . . . . . . . 48

4.3 Resultados numéricos obtidos pelo Método de Crank-Nicolson na aproximação da solução do problema (4.6). . . . . . . . . . 49

4.4 Resultados numéricos obtidos pela Técnica do Super-Passo na aproximação da solução do problema (4.6) . . . . . . . . . . . . 50

4.5 Erros cometidos nas aproximações da solução da equação (4.7). 52

4.6 Comparação entre os pseudo-códigos da Técnica do SuperPasso e do Método de Euler. . . . . . . . . . . . . . . . . . 54 


\section{Introdução}

A modelagem matemática de problemas físicos freqüentemente envolve a resolução de equações diferenciais parciais. Em particular, a condução de calor num meio físico pode ser modelada por uma equação diferencial parcial do tipo parabólico.

Quando não é possível explicitar a solução da equação diferencial parcial utilizam-se métodos numéricos para aproximar a solução do modelo. Neste trabalho utilizaram-se métodos implícitos e explícitos baseados em diferenças finitas. Os métodos explícitos restringem a escolha do passo de integração no tempo $\Delta t$ em função do passo de integração espacial $\Delta x$. Essa restrição é conhecida como condição de estabilidade e, em geral, os métodos implícitos ou não a possuem ou a apresentam de forma muito mais branda. Maiores detalhes podem ser encontrados em [3], [5], [4], [8], [14], [19], [22] e [25]. A condição de estabilidade impõe aos métodos explícitos uma maior quantidade de passos no tempo do que os métodos implícitos, tornando-os menos eficientes computacionalmente, entretanto como o tamanho do passo no tempo dos métodos implícitos é maior do que os permitidos pela condição de estabilidade dos métodos explícitos, os erros cometidos na aproximação da solução do problema proposto também são maiores. É uma situação onde a relação custo benefício deve ser analisada.

A Técnica do Super-Passo aplicada a algum método explícito permite a escolha do passo no tempo maior do que o permitido pela condição de esta- 
Introdução

bilidade. O algoritmo do Super-Passo é baseado no relaxamento da condição de estabilidade em cada passo no tempo, para impô-la apenas ao final de um ciclo de $N$ passos intermediários auxiliares. O esquema aqui apresentado é uma variação do Método de Gretzsch [13]. A implementação da Técnica do Super-Passo é simples; basta uma pequena modificação no código que emprega o método explícito para que o tempo de processamento seja até da ordem de 10 vezes menor, como pode ser visto em [2], [1], [10], [18], [20], [21] e [29].

O presente trabalho mostra que a Técnica do Super-Passo aplicada ao Método de Euler Explícito tem o tempo de processamento e erro cometido na aproximação similares aos obtidos pelo Método de Euler Implícito. Foram realizadas comparações entre os erros cometidos na aproximação da solução e tempo de processamento para os Métodos de Euler Explícito, Implícito, Crank-Nicolson e Super-Passo aplicado ao Método de Euler Explícito.

No primeiro capítulo é realizada uma introdução às equações diferenciais parciais, as equações são classificadas em parabólicas, hiperbólicas e elípticas e na última seção do capítulo é deduzida, a partir fenômenos físicos, a equação do calor com condutibilidade variável. Maiores detalhes sobre as equações diferenciais parciais podem ser encontrados em [9], [15], [16] e [17].

O Capítulo 2 apresenta a discretização da equação do calor com condutibilidade variável utilizando diferenças finitas e, para esta equação são, empregados os Métodos de Euler Explícito, Implícito e Crank-Nicolson e, para cada método numérico, são realizados os cálculos necessários para se obter as condições de estabilidade e consistência. Os métodos numéricos apresentados neste capítulo podem ser encontrados com uma abordagem mais ampla em [3], [5], [4], [8], [14], [19], [22] e [25].

O Terceiro Capítulo apresenta a construção da Técnica do Super-Passo aplicada ao Método de Euler Explícito, as condições de estabilidade, consistência e erro cometido na aproximação numérica da solução da equação 
do calor por esta técnica. A construção e aplicações do Super-Passo podem ser encontrados em [2], [1], [10], [18], [20], [21] e [29].

No Capítulo 4 é feita a validação dos Métodos de Euler Explícito, Implícito, Crank-Nicolson e da Técnica Super-Passo por refinamento de malha e apresenta os resultados obtidos pelos métodos ao aproximar a solução da equação do calor com condutibilidade variável.

O quinto capítulo apresenta a conclusão do trabalho e sugestões para a continuidade do mesmo.

No apêndice é realizada uma revisão sobre a teoria dos polinômios de Chebyshev, são apresentadas suas formulações, raízes e algumas propriedades que foram utilizadas no presente trabalho. Maiores detalhes sobre os polinômios de Chebyshev podem ser encontrados em [12], [19], [24] e [28]. 


\section{Capítulo 1}

\section{Modelagem Matemática da Condução do Calor}

\subsection{Equações Diferenciais Parciais}

A modelagem matemática de problemas físicos freqüentemente resulta na formulação de uma Equação Diferencial Parcial (ou de um sistema de equações diferenciais parciais) que é uma equação envolvendo duas ou mais variáveis independentes $x_{1}, x_{2}, \ldots, x_{n}$ e derivadas parciais da função $u=$ $u\left(x_{1}, x_{2}, \ldots, x_{n}\right)$. A forma geral de uma equação diferencial parcial de ordem $k$ é dada por

$$
\Psi\left(x_{1}, x_{2}, \ldots, x_{n}, u, \ldots, \frac{\partial u}{\partial x_{1}}, \ldots, \frac{\partial u}{\partial x_{n}}, \frac{\partial^{2} u}{\partial x_{1}^{2}}, \frac{\partial^{2} u}{\partial x_{1} \partial x_{2}}, \ldots, \frac{\partial^{k} u}{\partial x_{n}^{k}}\right)=0
$$

No caso de um sistema de equações, $\Psi$ é uma função vetorial e $u$ um vetor das variáveis dependentes. Considerando apenas as equações de segunda 
ordem com duas variáveis $x$ e $t$, tem-se

$$
a \frac{\partial^{2} u}{\partial x^{2}}+2 b \frac{\partial^{2} u}{\partial x \partial t}+c \frac{\partial^{2} u}{\partial t^{2}}=\varphi\left(x, t, u, \frac{\partial u}{\partial x}, \frac{\partial u}{\partial t}\right)
$$

onde $a, b$ e $c$ são funções de $x, t, u, u_{x}, u_{t}, \operatorname{com} u_{x}=\frac{\partial u}{\partial x}, u_{t}=\frac{\partial u}{\partial t}$ e $u(x, t)$ a solução de (1.1). Em outra notação,

$$
a u_{x x}+2 b u_{x t}+c u_{t t}=\varphi\left(x, t, u, u_{x}, u_{t}\right),
$$

onde $a, b$ e $c$ são funções de $x, t, u, u_{x}, u_{t}, \operatorname{com} u_{x}=\frac{\partial u}{\partial x}, u_{t}=\frac{\partial u}{\partial t}$ e $u(x, t)$ a solução de (1.1).

Se $a \equiv b \equiv c \equiv 0$ então a equação é de primeira ordem. Quando em (1.1) $a, b$ e $c$ são funções de $x, t$ e $u$ apenas ela é dita quase-linear; se, adicionalmente, $\varphi$ não depender de $u_{x}$ e $u_{t}$ então é dita semi-linear; a equação será linear se $a, b, c$ e $\varphi$ forem funções apenas de $x$ e $t$. Em quaisquer outras situações, a equação será não-linear.

Uma equação diferencial parcial como em (1.1) pode possuir uma família de soluções, uma solução única ou ainda não possuir nenhuma solução.

\subsection{Classificação}

Nesta Seção são classificadas as equações diferenciais parciais quase-lineares de segunda ordem com duas variáveis independentes e coeficientes reais.

Considere apenas os termos com derivadas de segunda ordem de uma equação diferencial parcial quase-linear, ou seja,

$$
L(u)=a \frac{\partial^{2} u}{\partial x^{2}}+2 b \frac{\partial^{2} u}{\partial x \partial t}+c \frac{\partial^{2} u}{\partial t^{2}}
$$


onde $a, b$ e $c$ são funções de $x, t, u, u_{x}$ e $u_{t}$, não simultaneamente nulos, e $u(x, t)$ a solução de (1.1). A classificação das equações diferenciais parciaisé feita por analogia com a teoria das cônicas no plano. O estudo das curvas planas de $2^{o}$ grau é simplificado pela redução da equação a sua forma normal através de uma mudança linear de coordenadas.

Como no estudo das cônicas, pode-se fazer uma mudança de variáveis que reduz (1.2) à sua forma canônica; a classificação de (1.2) é feita analisando o sinal do discriminate $b^{2}-a c$. A mudança de variáveis que transforma a equação (1.2) em sua forma canônica é apresentada em [17], [16] e mais detalhadamente em [9]. Assim, o discriminante $b^{2}-4 a c$ define o tipo da equação diferencial parcial (1.2) em

- elíptica se $b^{2}-4 a c<0$;

- parabólica se $b^{2}-4 a c=0$;

- hiperbólica se $b^{2}-4 a c>0$.

A equação $u_{t}-\mu \Delta u=0$ com $(\mu>0)$ é do tipo parabólico e conhecida como equação do calor e modela a condução de calor em meios contínuos; $\Delta u=0$ é do tipo elíptico e denominada equação de Laplace e modela fenômenos estacionários; $u_{t t}-c^{2} u_{x x}=0$ é do tipo hiperbólico e conhecida como equação da onda e pode modelar a propagação de ondas num meio contínuo.

\subsection{Modelagem Matemática da Propagação de Calor}

A modelagem apresentada nesta seção pode ser encontrada mais detalhadamente em [11]. 
Considere uma barra de comprimento $\ell$, com área de secção $A$, feita de um material condutor uniforme de calor. Suponha que a superfície lateral da barra esteja isolada termicamente de modo a não permitir, através dela, transferências de calor com o meio ambiente. Transferências podem, entretanto, ocorrer através das extremidades da barra.

A uniformidade do material e o isolamento térmico lateral implicam que o fluxo de calor se dê somente na direção longitudinal, e, portanto, tem-se um problema de condução de calor em uma dimensão apenas.

Considere que $u(x, t)$ represente a temperatura em um ponto $x$ desta barra no instante $t$, onde $0 \leq x \leq \ell$ e $0 \leq t \leq T$. Fixados dois pontos $x_{0} \mathrm{e}$ $x_{0}+\delta$ com $0<\delta$ na barra, têm-se as respectivas temperaturas $T_{1}=u\left(x_{0}, t\right)$ e $T_{2}=u\left(x_{0}+\delta, t\right)$ para um dado instante de tempo $t$. Suponha que o fluxo de calor seja de $T_{1}$ para $T_{2}$, ou seja, $T_{1}>T_{2}$. A Figura (1.1) ilustra a propagação do calor para esta barra.

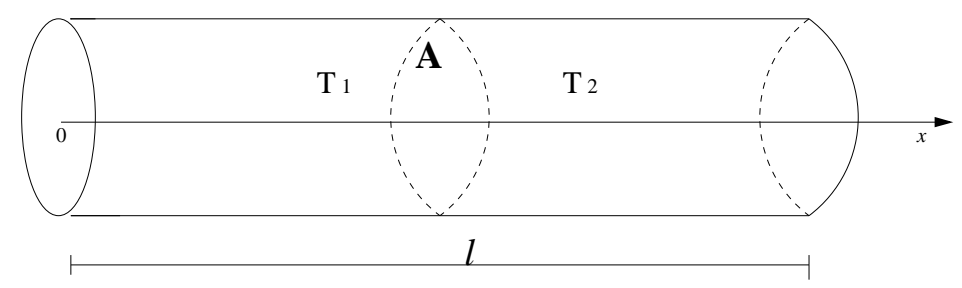

Figura 1.1: Propagação do calor em uma barra.

Pela Lei de Condução de Calor de Fourier, segue que

$$
Q=\mu A \frac{\left|T_{2}-T_{1}\right|}{\delta}
$$

onde $\mu$ é a condutibilidade térmica do material da barra, $\mu$ tem dimensão $W / m K$, ou seja, watts / metros Kelvin e $Q$, a quantidade de calor, é dada em Joules, J. 
Suponha que o limite $\lim _{\delta \rightarrow 0} Q=\mu A\left|u_{x}(x, t)\right|$ exista. Logo, o fluxo de calor entre as secções $x_{0}$ e $x_{0}+\delta$ pode ser expresso por

$$
q(x, t)=-\mu A u_{x}(x, t) .
$$

Se a condutibilidade térmica $\mu$ for dada em função em $x$, ou seja, $\mu=\mu(x)$, então o fluxo de calor entre as secções será dado por

$$
q(x, t)=-A \mu(x) u_{x}(x, t)
$$

Fixado um elemento da barra entre $\left[x_{0}\right.$ e $\left.x_{0}+\delta\right]$ a quantidade de calor, $q$, contida neste elemento da barra em um período de tempo entre $t_{0}$ e $t_{0}+\tau$ é expressa por

$$
q=\int_{t_{0}}^{t_{0}+\tau} q\left(x_{0}, t\right) d t-\int_{t_{0}}^{t_{0}+\tau} q\left(x_{0}+\delta, t\right) d t .
$$

Substituindo (1.3) na expressão (1.4) segue que

$$
q=\int_{t_{0}}^{t_{0}+\tau} A\left[\mu\left(x_{0}+\delta\right) u_{x}\left(x_{0}+\delta, t\right)-\mu\left(x_{0}\right) u_{x}\left(x_{0}, t\right)\right] d t
$$

Por outro lado, utilizando a densidade $\rho(x)$, a quantidade de calor pode ser determinada da seguinte maneira:

$$
\int_{t_{0}}^{t_{0}+\tau} \int_{x_{0}}^{x_{0}+\tau} c \rho(x) A u_{t}(x, t) d x d t,
$$

onde $c$ é o calor específico do material, dado em $J / k g K$, ou seja, Joules / kilograma Kelvin e $\rho(x)$ a densidade do material que a barra é feita e é dado em kilograma / metro cúbico, $\mathrm{kg} / \mathrm{m}^{3}$.

Aplicando o Teorema Fundamental do Cálculo em (1.5) e igualando a (1.6) tem-se 


$$
\int_{t_{0}}^{t_{0}+\tau} \int_{x_{0}}^{x_{0}+\tau}\left[\mu(x) u_{x}(x, t)\right]_{x} d x d t=\int_{t_{0}}^{t_{0}+\tau} \int_{x_{0}}^{x_{0}+\tau} \rho(x) A u_{t}(x, t) d x d t
$$

Assim, segue que

$$
c \rho(x) u_{t}(x, t)=\left[\mu(x) u_{x}(x, t)\right]_{x},
$$

onde $\rho(x)$ e $\mu(x)$ são a densidade e a condutibilidade térmica da barra, respectivamente, e $c$ o calor específico. Note que $\rho(x), \mu(x)>0, \forall x \in \Re$.

A equação (1.7) é a equação do calor com coeficientes variáveis para uma barra definida no domínio $\Omega=\left\{(x, t) \in \Re^{2} / 0 \leq x \leq \ell\right.$ e $\left.\quad 0 \leq t \leq T\right\}$, com $\ell, T$ positivos fixos.

A equação (1.7), da forma que está definida, não garante a unicidade da solução $u(x, t)$. Para que a equação possua solução são necessárias informações adicionais sobre a função $u(x, t)$ conhecidas como condição inicial e condições de contorno, maiores detalhes pode ser encontrados em [9], [11], [15], [16] e [17].

Considere a equação diferencial parcial do tipo parabólico dada em (1.7) em conjunto com as seguintes condições de contorno e condição inicial:

$$
\left\{\begin{array}{l}
\rho(x) u_{t}(x, t)=\left[\mu(x) u_{x}(x, t)\right]_{x} \\
u(x, 0)=\phi(x), \quad x \in[0, \ell] \\
u(0, t)=g(t), \quad t \in[0, T] \\
u(\ell, t)=h(t), \quad t \in[0, T]
\end{array}\right.
$$

onde $\rho(x), \mu(x)>0 \forall x \in[0, \ell]$, com $\mu(x)$ derivável em $[0, \ell]$, e $g(t), h(t)$ contínuas em $[0, T]$ e $\phi(x)$ contínua em $[0, \ell]$. Impondo ainda que as funções $g(t), h(t)$ e $\phi(x)$ sejam compatíveis, ou seja, $\phi(0)=g(0)$ e $\phi(\ell)=h(0)$, o 
teorema a seguir garante a existência e unicidade da solução do problema (1.8).

Teorema 1 Seja u contínua em $\bar{\Omega}$, suas derivadas contínuas em $\Omega$. Então $u$ é unicamente determinada em $\bar{\Omega}$ pelos valores de $u$ em $\partial \Omega$.

No Teorema $1 \bar{\Omega}$ representa o interior do conjunto $\Omega$, e $\partial \Omega$ a sua fronteira, a demosntração deste teorema encontra-se em [17] e [9].

Pelo teorema acima conclui-se que o problema proposto em (1.8) possui solução única no domínio $\Omega$, pois as condições de contorno e iniciais são contínuas e coincidem com a solução da equação diferencial parcial na fronteira de $\Omega$. 


\section{Capítulo 2}

\section{Métodos Numéricos Clássicos}

Neste Capítulo são apresentados os métodos de Euler Explícito, Implícito e Crank-Nicolson. A condição de estabilidade para cada método também é determinada e mostra-se que os métodos numéricos obtidos são consistentes com a equação diferencial parcial do tipo parabólico em (1.7) com densidade constante. Por simplicidade, tomar-se-á $c=1$ e $\rho(x) \equiv 1$, para todo $x$, e um termo forçante $f(x, t)$ de classe $C^{\infty}$.

\subsection{Discretização: Diferenças Finitas}

Considere o seguinte problema de Dirichilet

$$
\left\{\begin{array}{l}
u_{t}(x, t)-\left[\mu(x) u_{x}(x, t)\right]_{x}=f(x, t) \\
u(x, 0)=\phi(x) \quad x \in[0, L] \\
u(0, t)=g(t) \quad t \in[0, T] \\
u(L, t)=h(t) \quad t \in[0, T]
\end{array}\right.
$$

definido no domínio $\Omega=[0, L] \times[0, T], \operatorname{com} \mu(x)>0, \forall x \in[0, L], f(x, t) \in$ $C^{\infty}(\Omega)$. 
O intervalo $[0, L]$, da variável espacial $x$, é dividido em $N$ partes iguais de comprimento $\Delta x$, obtendo-se $N+1$ pontos $x_{n}=n \Delta x, n=0,1, \ldots, N$, onde $\Delta x=\frac{L}{N}$. O instante de tempo final $T$ do intervalo $[0, T]$ da variável temporal $t$ é dividido pelo passo de integração no tempo $\Delta t$, determinado respeitando (quando existe) a condição de estabilidade do método numérico, obtendo-se a quantidade de passos no tempo $M$, ou seja, tem-se $M+1$ pontos $t_{m}=m \Delta t, m=0,1, \ldots, M$. Assim, procura-se aproximar a solução da equação diferencial nos pontos da malha $\left(x_{n}, t_{m}\right)$, ilustrada na Figura (2.1).

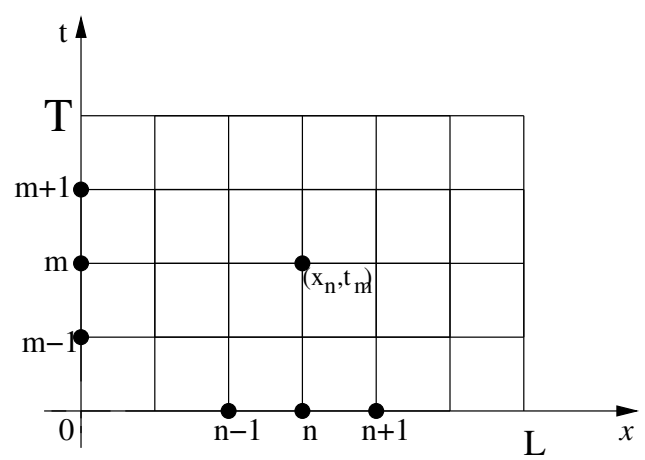

Figura 2.1: Discretização do domínio $\Omega$.

O valor da solução exata no ponto $\left(x_{n}, t_{m}\right)$ será representado por $u_{n}^{m}$ e a aproximação obtida por um método numérico por $U_{n}^{m}$.

O problema (2.1) é discretizado utilizando diferenças finitas, ou seja, para aproximar a derivada de $1^{a}$ ordem de $u(x, t)$ em relação a $t$ utilizam-se as seguintes aproximações:

$$
\begin{gathered}
u_{t}(x, t) \approx \frac{u(x, t+\Delta t)-u(x, t)}{\Delta t} \quad \text { (Fórmula Progressiva) }, \\
u_{t}(x, t) \approx \frac{u(x, t)-u(x, t-\Delta t)}{\Delta t} \quad \text { (Fórmula Regressiva). }
\end{gathered}
$$

Para aproximar a derivada de $2^{a}$ ordem da função $u(x, t)$ em relação a $t$, 
utiliza-se a seguinte aproximação:

$$
u_{t t}(x, t) \approx \frac{u(x, t-\Delta t)-2 u(x, t)+u(x, t+\Delta t)}{\Delta t^{2}} .
$$

As aproximações acima são oriundas da manipulação algébrica da expansão em Série de Taylor da função $u$ em $(x, t+\Delta t)$ :

$$
u(x, t \pm \Delta t)=u \pm \Delta t \frac{\partial u}{\partial t}+\frac{\Delta x^{2}}{2 !} \frac{\partial^{2} u}{\partial t^{2}} \pm \frac{\Delta x^{3}}{6 !} \frac{\partial^{3} u}{\partial t^{3}}+O\left(\Delta t^{4}\right)
$$

Por intermédio da manipulação algébrica das aproximações (2.2), (2.3) e (2.4) é possível construir os Métodos de Euler Explícito, Euler Implícito e Crank-Nicolson, essas construções são detalhadas em [3], [4], [5] e [8].

Considere uma equação diferencial parcial de segunda ordem definida no domínio $\Omega$.

$$
P(u)=f\left(x, t, u, u_{t}, u_{x}\right)
$$

onde $P(u)$ é dada por,

$$
P(u)=a(x, t) u_{x x}+b(x, t) u_{x t}+c(x, t) u_{t t}+d(x, t) u_{t}+e(x, t) u_{x},
$$

$\operatorname{com} a, b, c, d, e, u$ e suas derivadas contínuas em $\Omega$. Um método de diferenças finitas com passo de integração no tempo $\Delta t$ e passo de integração no espaço $\Delta x$ para (2.6) é denotado por $P_{\Delta}(U)$, as definições a seguir são discutidadas em $[25]$.

Definição 1 Um método de diferenças finitas $P_{\Delta} U_{n}^{m}=0$ para uma equação de primeira ordem é estável numa região de estabilidade $\Lambda$ se existir um inteiro $J$ tal que para qualquer instante de tempo positivo $T$, existir uma constante $C_{T}$ tal que 


$$
\Delta x \sum_{j=-\infty}^{\infty}\left|U_{n}^{m}\right|^{2} \leq C_{T} \Delta x \sum_{j=0}^{J} \sum_{i=-\infty}^{\infty}\left|U_{i}^{j}\right|^{2},
$$

para $0 \leq m \Delta t \leq T, \operatorname{com}(\Delta x, \Delta t) \in \Lambda$.

A estabilidade é uma condição necessária para a convergência do método numérico, uma das formas funcionais de se determinar a condição de estabilidade de um método numérico de passo único no tempo é a Análise de Von Neumann, que é ilustrada a seguir e, detalhada em [25].

\section{Análise de Von Neumann}

Uma importante aplicação das Transformadas de Fourier é a Análise de Von Neumann para a determinação da condição de estabilidade de métodos numéricos baseados em diferenças finitas. As Transformadas de Fourier fornecem condições suficientes e necessárias para a estabilidade dos métodos de diferenças finitas.

POr intermédio da Transformada de Fourier a determinação da condição de estabilidade de um método de diferenças é reduzido a manipulações algébricas. Considere a equação $u(x, t)_{t}=-\mu u(x, t)_{x}$, com $0<a$ e $u(x, t) \in C^{2}(\Omega)$, onde $\Omega$ é o domínio da função $u(x, t)$, e o método de diferenças dado a seguir:

$$
\frac{U_{n}^{m+1}-U_{n}^{m}}{\Delta t}=-\mu \frac{U_{n}^{m}-U_{n}^{m-1}}{\Delta x}
$$

que pode ser reescrito como

$$
U_{n}^{m+1}=\left(1-\mu \frac{\Delta t}{\Delta x}\right) U_{n}^{m}+\mu \frac{\Delta t}{\Delta x} U_{n-1}^{m} .
$$

Aplicando a Transformada Inversa de Fourier para $U_{n}^{m}$, tem-se

$$
U_{n}^{m}=\frac{1}{\sqrt{2 \pi}} \int_{-\pi / \Delta x}^{\pi / \Delta x} e^{i n \Delta x \xi} \hat{U}^{m}(\xi) d \xi
$$


substituindo em (2.9) para $U_{n}^{m}$ e $U_{n-1}^{m}$, obtém-se

$$
U_{n}^{m+1}=\frac{1}{\sqrt{2 \pi}} \int_{-\pi / \Delta x}^{\pi / \Delta x} e^{i n \Delta x \xi}\left[\left(1-\mu \frac{\Delta t}{\Delta x}\right)+\mu \frac{\Delta t}{\Delta x} e^{-i \Delta x \xi}\right] \hat{U}^{m}(\xi) d \xi
$$

Comparando a expressão (2.10) com a transformada Inversa de Fourier

$$
U_{n}^{m+1}=\frac{1}{\sqrt{2 \pi}} \int_{-\pi / \Delta x}^{\pi / \Delta x} e^{i n \Delta x \xi} \hat{U}^{m+1}(\xi) d \xi
$$

e usando o fato que a Transformada de Fourier é única, segue que o integrando de (2.10) é igual ao da Transforma Inversa de Fourier, assim tem-se que

$$
\hat{U}^{m+1}(\xi)=\left[\left(1-\mu \frac{\Delta t}{\Delta x}\right)+\mu \frac{\Delta t}{\Delta x} e^{i \Delta x \xi}\right] \hat{U}^{m}(\xi)=g(\Delta x \xi) \hat{U}^{m}(\xi)
$$

onde

$$
g(\Delta x \xi)=\left(1-\mu \frac{\Delta t}{\Delta x}\right)+\mu \frac{\Delta t}{\Delta x} e^{i \Delta x \xi} .
$$

A expressão2.11 mostra que realizar um passo de integração no tempo do método numérico é equivalente a multiplicar a Transformada de Fourier da solução pelo fator de amplificação $g(\Delta x \xi)$. Assim, obtém-se a relação

$$
\hat{U}^{m}(\xi)=g^{m}(\Delta x \xi) \hat{U}^{0}(\xi) .
$$

Note que o índice $m$ em $\hat{U}$ indica quantidade de passos de integração no tempo, enquanto em $g$ é uma potência. De modo a ilustrar os cálculos necessários para se determinar a condição de estabilidade de um dado método numérico, determinemos a condição de estabilidade do método de diferenças dado em (2.8). Utilizando a expressão (2.12) e a Identidade de Parseval em (2.8), tem-se 


$$
\begin{aligned}
\Delta x \sum_{j=-\infty}^{\infty}\left|U_{j}^{m}\right|^{2} & =\int_{-\pi / \Delta x}^{\pi / \Delta x}\left|\hat{U}^{m}(\xi)\right|^{2} d \xi \\
& =\int_{-\pi / \Delta x}^{\pi / \Delta x}|g(\Delta x \xi)|^{2 m}\left|\hat{U}^{0}(\xi)\right|^{2} d \xi .
\end{aligned}
$$

A desigualdade em (2.7) será satisfeita, com $J=0$, se $|g(\Delta x \xi)|^{2 m}$ for apropriadamente limitada. Considere $\theta=\Delta x \xi$, então o fator de amplificação para o método (2.8) é dado por

$$
\begin{aligned}
|g(\theta)|^{2} & =\left|\left(1-\mu \frac{\Delta t}{\Delta x}\right)+\mu \frac{\Delta t}{\Delta x} e^{-i \theta}\right|=\left(1-\mu \frac{\Delta t}{\Delta x}+\mu \frac{\Delta t}{\Delta x} \cos (\theta)\right)^{2}+\mu^{2}\left(\frac{\Delta t}{\Delta x}\right)^{2} \sin ^{2}(\theta) \\
& =\left(1-2 \mu \frac{\Delta t}{\Delta x} \sin ^{2}\left(\frac{\theta}{2}\right)\right)^{2}+4 \mu^{2}\left(\frac{\Delta t}{\Delta x}\right)^{2} \sin ^{2}\left(\frac{\theta}{2}\right) \cos ^{2}\left(\frac{\theta}{2}\right) \\
& =1-4 \mu \frac{\Delta t}{\Delta x} \sin ^{2}\left(\frac{\theta}{2}\right)+4 \mu^{2}\left(\frac{\Delta t}{\Delta x}\right)^{2} \sin ^{4}\left(\frac{\theta}{2}\right)+4 \mu^{2}\left(\frac{\Delta t}{\Delta x}\right)^{2} \sin ^{2}\left(\frac{\theta}{2}\right) \cos ^{2}\left(\frac{\theta}{2}\right) \\
& =1-4 \mu \frac{\Delta t}{\Delta x}\left(1-\mu \frac{\Delta t}{\Delta x}\right) \sin ^{2}\left(\frac{\theta}{2}\right) .
\end{aligned}
$$

Note que $|g(\theta)|$ é limitado por 1 se $0 \leq \mu \frac{\Delta t}{\Delta x} \leq 1$. Assim, por (2.12), segue que

$$
\Delta x \sum_{j=-\infty}^{\infty}\left|U_{j}^{m}\right|^{2} \leq \int_{-\pi / \Delta x}^{\pi / \Delta x}\left|\hat{U}^{0}(\xi)\right|^{2} d \xi=\Delta x \sum_{j=-\infty}^{\infty}\left|U_{j}^{0}\right|^{2} .
$$

Pela definição (2.7) o método é estável. Entretanto, se o número $\mu \frac{\Delta t}{\Delta x}$ não estiver fixado entre 0 e 1 , quando $\Delta x$ e $\Delta t$ tenderem a zero, então $|g(\theta)|$ será maior do que 1 para alguns valores de $\theta$, e o método será instável.

No estudo da condição de estabilidade dos métodos numéricos adotou-se a notação empregada em (2.12). 
Teorema 2 Um método de diferenças finitas de passo único (com coeficientes constantes) é estável, se e somente se, existir uma constante $K$ (independente de $\theta, \Delta x$ e $\Delta t$ ) e alguns passos de integração $\Delta t_{0}$ e $\Delta x_{0}$ tais que

$$
|g(\theta, \Delta x, \Delta t)| \leq 1+K \Delta t
$$

para todo $\theta, 0<\Delta t \leq \Delta t_{0}$, e $0<\Delta x \leq \Delta x_{0}$.

Se $g(\theta, \Delta x, \Delta t)$ for independente de $\Delta x$ e $\Delta t$, a condição de estabilidade (2.13) pode ser substituída por

$$
|g(\theta)| \leq 1
$$

Este teorema mostra que para se determinar a condição de estabilidade de um método de diferenças finitas considera-se apenas o fator de amplificação $g(\Delta x \xi)$ oriundo da análise de Von Neumann, este teorema, e as definições 2 e 3 são discutidas em [25].

Definição 2 Dada uma equação diferencial parcial $P(u)$ definida num domínio $\Omega$ e um método de diferenças finitas, $P_{\Delta}(U)$, com passos de integração no tempo e espaço dados por $\Delta t$ e $\Delta x$. O método de diferenças finitas é dito consistente com a equação diferencial parcial se para qualquer função suave $\phi(x, t)$

$$
\left|P \phi(x, t)-P_{\Delta} \phi(x, t)\right| \rightarrow 0 \quad \text { quando } \quad \Delta x, \Delta t \rightarrow 0, \quad \forall(x, t) \in \Omega
$$

A consistência é uma condição necessária para a convergência do método numérico, pois, garante que o método de diferenças finitas aproxima a equação diferencial quando os passos de integração no tempo tendem a zero.

Definição 3 Um método de diferenças finitas de passo único que aproxima uma equação diferencial parcial é dito convergente, se para quaisquer solução 
$u(x, t)$, da equação diferencial e $U_{n}^{m}$ a aproximação gerada pelo método numérico, tais que,

$$
U_{n}^{0} \longrightarrow u(x, 0), \quad \text { quando } n \Delta x \longrightarrow x
$$

então

$$
U_{n}^{m} \longrightarrow u(x, t), \quad \text { quando } \quad(n \Delta x, m \Delta t) \longrightarrow(x, t),
$$

com

$$
\Delta x, \Delta t \longrightarrow 0
$$

O Teorema de Lax-Richtmyer enuncia um resultado necessário e suficiente para a convergência de métodos de diferenças finitas.

Teorema 3 Teorema de equivalência de Lax-Richtmyer Um método consistente de diferenças finitas para uma equação diferencial parcial é convergente se e somente se o método for estável.

O Teorema de equivalência de Lax é demostrado em [25], as definições acima são encontradas em [8], [25].

\subsection{Método de Euler Explícito}

Na discretização da equação (2.1) utilizou-se a seguinte aproximação para a derivada de primeira ordem no espaço:

$$
u_{x}\left(x_{n}, t_{m}\right) \simeq \frac{u_{n+\frac{1}{2}}^{m}-u_{n-\frac{1}{2}}^{m}}{\Delta x},
$$

onde $\quad u_{n+\frac{1}{2}}^{m}=u\left(x_{n+\frac{1}{2}}, t_{m}\right)$.

Aplicando essa aproximação na parte espacial da equação (2.1) e aproximando a derivada de primeira ordem no tempo por diferenças progressivas 
no tempo (2.2) obtém-se:

$$
\frac{U_{n}^{m+1}-U_{n}^{m}}{\Delta t}-\frac{\mu_{n+\frac{1}{2}}\left(U_{n+1}^{m}-U_{n}^{m}\right)-\mu_{n-\frac{1}{2}}\left(U_{n}^{m}-U_{n-1}^{m}\right)}{\Delta x^{2}}=f_{n}^{m},
$$

ou seja,

$$
U_{n}^{m+1}=U_{n}^{m}+\frac{\Delta t}{\Delta x^{2}}\left[\mu_{n+\frac{1}{2}}\left(U_{n+1}^{m}-U_{n}^{m}\right)-\mu_{n-\frac{1}{2}}\left(U_{n}^{m}-U_{n-1}^{m}\right)\right]+\Delta t f_{n}^{m}
$$

onde $\mu_{n+\frac{1}{2}}=\mu\left(x_{n}+\frac{\Delta x}{2}\right)$, e $n=1,2 \ldots, N-1$ e $m=0,1 \ldots, M-1$.

Lembrando que o intervalo espacial $[0, L]$ possui $N+1$ pontos e o intervalo temporal $[0, T]$ contém $M+1$ pontos.

No caso especial em que a condutibilidade térmica $\mu$ é constante, a expressão (2.15) pode ser escrita como

$$
U_{n}^{m+1}=U_{n}^{m}+\mu \frac{\Delta t}{\Delta x^{2}}\left(U_{n+1}^{m}-2 U_{n}^{m}+U_{n-1}^{m}\right)+\Delta t f_{n}^{m},
$$

$\operatorname{com} n=1,2 \ldots, N-1$ e $m=0,1 \ldots, M-1$.

A Figura (2.2) ilustra a molécula computacional da fórmula (2.16) que é conhecida como Método de Euler Explícito.

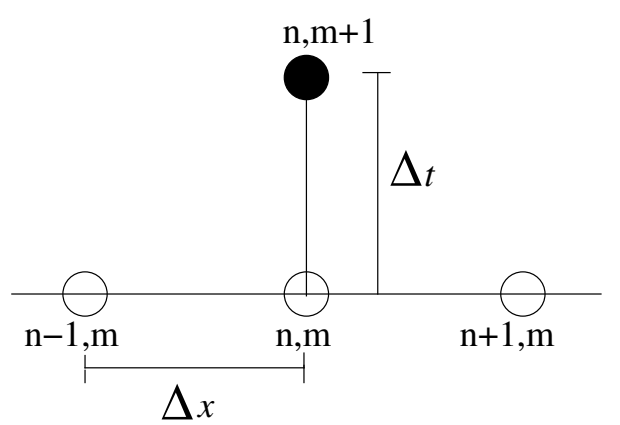

Figura 2.2: Molécula computacional do Método de Euler Explícito.

A Figura (2.2) ilustra a dependência que a aproximação obtida no ponto $\left(x_{n}, t_{m+1}\right)$ tem em relação aos pontos $\left(x_{n-1}, t_{m}\right),\left(x_{n}, t_{m}\right)$ e $\left(x_{n+1}, t_{m}\right)$, ou seja, 
é necessário conhecer os valores $U_{n-1}^{m}, U_{n}^{m}$ e $U_{n+1}^{m}$ para que a aproximação $U_{n}^{m+1}$ possa ser calculada, a construção deste método numérico pode ser encontrada em [3], [4], [5] e [8].

\section{Condição de Estabilidade do Método de Euler Explícito}

Considere o problema proposto em (2.1), com o domínio discretizado e o Método de Euler Explícito descrito em (2.16) e suponha que $f(x, t) \equiv 0$.

No Critério de Von Neumann, para determinar estabilidade do esquema, examina-se a propagação do erro global, tendo em vista que ele é a soma de erros mais simples chamados de harmônicos. O processo é inspirado na expansão de uma função em série de Fourier. Substitui-se $u_{n}^{m}$ por $g^{m} e^{i n \theta} \mathrm{em}$ (2.16), e adotando $\mu_{M}=\max _{0 \leq x \leq L}\{\mu(x)\}$, tem-se:

$$
\begin{gathered}
g^{m+1} e^{i n \theta}=g^{m} e^{i n \theta}+\frac{\Delta t}{\Delta x^{2}} \mu_{M}\left(g^{m} e^{i(n+1) \theta}-2 g^{m+1} e^{i n \theta}+g^{m} e^{i(n-1) \theta}\right) \Rightarrow \\
g g^{m} e^{i n \theta}=g^{m} e^{i n \theta}+\frac{\Delta t}{\Delta x^{2}} \mu_{M}\left(g^{m} e^{i n \theta} e^{i \theta}-2 g^{m} e^{i n \theta}+g^{m} e^{i n \theta} e^{-i \theta}\right),
\end{gathered}
$$

$\log 0$

$$
g=1+\frac{\Delta t}{\Delta x^{2}} \mu_{M}\left(e^{i \theta}-2+e^{-i \theta}\right)
$$

utilizando a identidade $e^{a+b i}=e^{a}(\cos (b)+i \sin (b))$ na expressão acima segue que:

$$
g=1+\frac{\Delta t}{\Delta x^{2}} \mu_{M}(2 \cos (\theta)-2)=1+2 \frac{\Delta t}{\Delta x^{2}} \mu_{M}(\cos (\theta)-1)
$$

pela seguinte relação trigonométrica $\cos (x)=1-2 \sin ^{2}\left(\frac{x}{2}\right)$ tem-se

$$
g=1-4 \frac{\Delta t}{\Delta x^{2}} \mu_{M} \sin ^{2}\left(\frac{\theta}{2}\right)
$$


Para que o método seja estável é necessário que haja amortecimento do erro, ou seja $|g| \leq 1$.

Assim,

$$
-1 \leq 1-4 \frac{\Delta t}{\Delta x^{2}} \mu_{M} \sin ^{2}\left(\frac{\theta}{2}\right) \leq 1
$$

donde, é necessário que

$$
\begin{aligned}
-1 & \leq 1-4 \frac{\Delta t}{\Delta x^{2}} \mu_{M} \sin ^{2}\left(\frac{\theta}{2}\right) \Rightarrow \\
2 & \geq 4 \frac{\Delta t}{\Delta x^{2}} \mu_{M} \sin ^{2}\left(\frac{\theta}{2}\right) \Rightarrow \\
\frac{1}{2} & \geq \frac{\Delta t}{\Delta x^{2}} \mu_{M} \sin ^{2}\left(\frac{\theta}{2}\right)
\end{aligned}
$$

Portanto, para que o Método de Euler Explícito seja estável é necessário que os parâmetros $\Delta x$ e $\Delta t$ atendam a seguinte condição de estabilidade:

$$
\frac{\Delta t}{\Delta x^{2}} \leq \frac{1}{2 \mu_{M}}
$$

onde $\mu_{M}=\max _{0 \leq x \leq L}\{\mu(x)\}$.

Note que esta restrição para o tamanho do passo na direção $t$ pode ser interpretada como $\Delta t<\frac{\Delta x^{2}}{2 \mu_{M}}$, assim, se a aproximação for feita para um valor de $T$ grande, o esforço computacional também será alto.

\section{Consistência do Método de Euler Explícito}

Suponha que $u(x, t)$ seja uma função suficientemente suave, $P u(x, t)$ a equação diferencial em (2.1) e $P_{\Delta} u$ a equação diferencial em (2.1) discretizada com o Método Euler Explícito, com $u_{n}^{m}=u\left(x_{n}, t_{m}\right)$ e $\mu_{M}=\max _{0 \leq x \leq L}\{\mu(x)\}$, então:

$$
P_{\Delta} u=\frac{u_{n}^{m+1}-u_{n}^{m}}{\Delta t}-\mu_{M} \frac{u_{n-1}^{m}-2 u_{n}^{m}+u_{n+1}^{m}}{\Delta x^{2}}
$$


Aplicando as expansões em série de Taylor (2.5) acima obtém-se:

$$
\begin{aligned}
P_{\delta} u & =\frac{u_{n}^{m}+\Delta t u_{t}+\frac{1}{2} \Delta t^{2} u_{t t}+O\left(\Delta t^{3}\right)-u_{n}^{m}}{\Delta t}-\mu_{M} \frac{u_{n-1}^{m}-2 u_{n}^{m}+u_{n+1}^{m}}{\Delta x^{2}}, \\
& =\frac{\Delta t u_{t}+O\left(\Delta t^{2}\right)}{\Delta t}-\mu_{M} \frac{\Delta x^{2} u_{x x}+O\left(\Delta x^{3}\right)}{\Delta x^{2}} \\
& =u_{t}+O(\Delta t)-\mu_{M}\left(u_{x x}+O(\Delta x)\right)
\end{aligned}
$$

$\log 0$

$$
P_{\Delta} u=u_{t}+O(\Delta t)-\mu_{M}\left(u_{x x}+O(\Delta x)\right),
$$

onde as derivadas são calculadas no ponto $\left(x_{n}, t_{m}\right)$, ou seja, $u_{t}=u_{t}\left(x_{n}, t_{m}\right)$. Considerando a diferença entre $P u$ e $P_{\Delta} u$ tem-se,

$$
\begin{gathered}
P u-P_{\Delta} u=u_{t}-\mu_{M} u_{x x}-u_{t}+O(\Delta t)+\mu_{M}\left(u_{x x}+O(\Delta x)\right), \\
P u-P_{\Delta} u=O(\Delta t)+\mu_{M} O(\Delta x) .
\end{gathered}
$$

Assim

$$
\lim _{\substack{\Delta t \rightarrow 0 \\ \Delta x \rightarrow 0}} P u-P_{\Delta} u=\lim _{\substack{\Delta t \rightarrow 0 \\ \Delta x \rightarrow 0}} O(\Delta t)+\mu_{M} O(\Delta x)=0 .
$$

Portanto, o Método de Euler Explícito é consistente com a equação diferencial.

Pelos resultados obtidos em (2.2), e aplicando o Teorema de Lax-Richtmyer, o Método de Euler Explícito é convergente. 


\subsection{Método de Euler Implícito}

A discretização de (2.1) foi realizada utilizando-se a seguinte aproximação para aderivada de primeira ordem,

$$
u_{x}\left(x_{n}, t_{m}\right) \simeq \frac{u_{n+\frac{1}{2}}^{m}-u_{n-\frac{1}{2}}^{m}}{\Delta x},
$$

onde $\quad u_{n+\frac{1}{2}}^{m}=u\left(x_{n+\frac{1}{2}}, t_{m}\right)$.

Aplicando na parte espacial da equação (2.1) e aproximando a derivada de primeira ordem no tempo por diferenças regressivas no tempo obtém-se,

$$
\frac{U_{n}^{m+1}-U_{n}^{m}}{\Delta t}-\frac{\mu_{n+\frac{1}{2}}\left(U_{n+1}^{m+1}-U_{n}^{m+1}\right)-\mu_{n-\frac{1}{2}}\left(U_{n}^{m+1}-U_{n-1}^{m+1}\right)}{\Delta x^{2}}=f_{n}^{m+1}
$$

agrupando as parcelas calculadas no mesmo instante de tempo tem-se

$-\frac{\Delta t}{\Delta x^{2}} \mu_{n+\frac{1}{2}} U_{n+1}^{m+1}+\left(1+\frac{\Delta t}{\Delta x^{2}}\left(\mu_{n+\frac{1}{2}}+\mu_{n-\frac{1}{2}}\right)\right) U_{n}^{m+1}-\frac{\Delta t}{\Delta x^{2}} \mu_{n-\frac{1}{2}} U_{n-1}^{m+1}=U_{n}^{m}+\Delta t f_{n}^{m+1}$,

onde $\mu_{n+\frac{1}{2}}=\mu\left(x_{n}+\frac{\Delta x}{2}\right)$, com $n=1,2, \ldots, N-1$ e $m=0,1, \ldots, M-1$.

O método descrito em (2.18) é chamado Método de Euler Implícito.

No caso em que $\mu(x)$ for uma função constante, a expressão (2.18) resumese a

$$
-\omega U_{n+1}^{m+1}+(1+2 \omega) U_{n}^{m+1}-\omega U_{n-1}^{m+1}=U_{n}^{m}+\Delta t f_{n}^{m+1},
$$

onde $\omega=\mu \frac{\Delta t}{\Delta x^{2}}$.

Note que, para obter a aproximação da solução no instante $t_{m+1}$, é necessária 
a resolução do seguinte sistema linear:

$$
A U_{m+1}=U_{m}+C_{m+1}+F_{m+1}, \quad m=0,1, \ldots, M-1,
$$

onde:

$$
\begin{gathered}
A=\left(\begin{array}{cccccc}
1+2 \omega & -\omega & 0 & \ldots & \ldots & 0 \\
0 & -\omega & 1+2 \omega & -\omega & \ldots & 0 \\
\vdots & & & & & \vdots \\
0 & \ldots & \ldots & 0 & -\omega & 1+2 \omega
\end{array}\right), \quad U_{m}=\left(\begin{array}{c}
U_{1}^{m} \\
U_{2}^{m} \\
\vdots \\
U_{N-2}^{m} \\
U_{N-1}^{m}
\end{array}\right) \\
C_{m+1}=\omega\left(\begin{array}{c}
U_{0}^{m+1} \\
0 \\
\vdots \\
0 \\
U_{N}^{m+1}
\end{array}\right), \quad F_{m+1}=\Delta t\left(\begin{array}{c}
f_{1}^{m+1} \\
f_{2}^{m+1} \\
\vdots \\
f_{N-2}^{m+1} \\
f_{N-1}^{m+1}
\end{array}\right),
\end{gathered}
$$

A molécula computacional do Método de Euler Implícito é ilustrada na Figura (2.3).

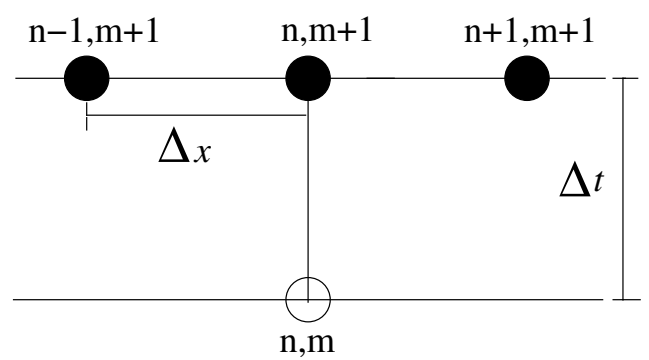

Figura 2.3: Molécula computacional do Método de Euler Implícito.

A Figura (2.3) ilustra a dependência que a aproximação obtida no ponto $\left(x_{n}, t_{m+1}\right)$ tem em relação aos pontos $\left(x_{n-1}, t_{m+1}\right),\left(x_{n}, t_{m}\right)$ e $\left(x_{n+1}, t_{m+1}\right)$, ou 
seja, é necessário conhecer os valores $U_{n-1}^{m+1}, U_{n}^{m}$ e $U_{n+1}^{m+1}$ para que a aproximação $U_{n}^{m+1}$ possa ser calculada, a construção do Método de Euler Implícito pode ser encontrada em [3], [4], [5] e [8].

\section{Condição de Estabilidade do Método de Euler Implícito}

Considere o problema proposto em (2.1), com domínio discretizado e o Método de Euler Implícito descrito em (2.18). Considerando $f(x, t) \equiv 0$ e $\mu_{M}=\max _{0 \leq x \leq L}\{\mu(x)\}$, substitui-se $u_{n}^{m}$ por $g^{m} e^{i n \theta}$ em (2.19), obtendo-se:

$$
\begin{gathered}
-\frac{\Delta t}{\Delta x^{2}} \mu_{M} g^{m+1} e^{i(n+1) \theta}+\left(1+2 \frac{\Delta t}{\Delta x^{2}} \mu_{M}\right) g^{m+1} e^{i n \theta}-\frac{\Delta t}{\Delta x^{2}} \mu_{M} g^{m+1} e^{i(n-1) \theta}=g^{m} e^{i n \theta} \Rightarrow \\
-\frac{\Delta t}{\Delta x^{2}} \mu_{M} g g^{m} e^{i n \theta} e^{i \theta}+\left(1+2 \frac{\Delta t}{\Delta x^{2}} \mu_{M}\right) g g^{m} e^{i n \theta}-\frac{\Delta t}{\Delta x^{2}} \mu_{M} g g^{m} e^{i n \theta} e^{-i \theta}=g^{m} e^{i n \theta},
\end{gathered}
$$

e, portanto,

$$
-\frac{\Delta t}{\Delta x^{2}} \mu_{M} g e^{i \theta}+\left(1+2 \frac{\Delta t}{\Delta x^{2}} \mu_{M}\right) g-\frac{\Delta t}{\Delta x^{2}} \mu_{M} g e^{-i \theta}=1
$$

Utilizando novamente a identidade $e^{a+b i}=e^{a}(\cos (b)+i \sin (b))$, segue que

$$
\begin{aligned}
-2 \frac{\Delta t}{\Delta x^{2}} \mu_{M} g \cos (\theta)+\left(1+2 \frac{\Delta t}{\Delta x^{2}} \mu_{M}\right) g & =1 \Rightarrow \\
g\left(2 \frac{\Delta t}{\Delta x^{2}} \mu_{M}(1-\cos (\theta))+1\right) & =1 .
\end{aligned}
$$

Aplicando a relação trigonométrica $\cos (x)=1-2 \sin ^{2}\left(\frac{x}{2}\right)$ na expressão acima, tem-se

$$
g\left(4 \frac{\Delta t}{\Delta x^{2}} \mu_{M} \sin ^{2}\left(\frac{\theta}{2}\right)\right)=1 .
$$

Logo,

$$
g=\frac{1}{4 \frac{\Delta t}{\Delta x^{2}} \mu_{M} \sin ^{2}\left(\frac{\theta}{2}\right)} .
$$


e segue que

$$
|g|<1 \quad \forall \theta
$$

Portanto, o Método de Euler Implícito é incondicionalmente estável, ou seja, independentemente do tamanho do passo de integração no tempo o Método de Euler Implícito não amplifica os erros cometidos na aproximação numérica da solução do equação diferencial.

\section{Consistência do Método de Euler Implícito}

Suponha que $u(x, t)$ seja uma função suficientemente suave, $P u(x, t)$ a equação diferencial e $P_{\Delta} u$ o Método Euler implícito, com $u_{n}^{m}=u\left(x_{n}, t_{m}\right)$ e $\mu_{M}=\max _{0 \leq x \leq L}\{\mu(x)\}$, então

$$
P_{\Delta} u=\frac{u_{n}^{m}-u_{n}^{m-1}}{\Delta t}-\mu_{M} \frac{u_{n-1}^{m}-2 u_{n}^{m}+u_{n+1}^{m}}{\Delta x^{2}} .
$$

Substituindo $u_{n}^{m+1}, u_{n-1}^{m}$ e $u_{n+1}^{m}$ pelas suas expansões em série de Taylor na equação (2.20) tem-se

$$
\begin{aligned}
P_{\Delta} u & =\frac{u_{n}^{m}-u_{n}^{m}+\Delta t u_{t}+O\left(\Delta t^{2}\right)}{\Delta t}-\mu_{M} \frac{-\Delta x u_{x}+\frac{\Delta x^{2}}{2} u_{x x}+\Delta x u_{x}+\frac{\Delta x^{2}}{2} u_{x x}+O\left(\Delta x^{3}\right)}{\Delta x^{2}} \\
& =u_{t}+O(\Delta t)-\mu_{M}\left(u_{x x}+O(\Delta x)\right),
\end{aligned}
$$

$\log \mathrm{O}$

$$
P_{\Delta} u=u_{t}-\mu_{M} u_{x x}+O(\Delta t)+O(\Delta x)
$$

onde as derivadas são calculadas no ponto $\left(x_{n}, t_{m}\right)$, ou seja, $u_{t}=u_{t}\left(x_{n}, t_{m}\right)$.

Considerando a diferença entre $P u$ e $P_{\Delta} u$ tem-se

$$
P u-P_{\Delta} u=u_{t}-\mu_{M} u_{x x}-u_{t}+\mu_{M} u_{x x}+O(\Delta t)+O(\Delta x),
$$


assim,

$$
P u-P_{\Delta} u=O(\Delta t)+O(\Delta x),
$$

segue que,

$$
\lim _{\substack{\Delta t \rightarrow 0 \\ \Delta x \rightarrow 0}} P u-P_{\Delta} u=\lim _{\substack{\Delta t \rightarrow 0 \\ \Delta x \rightarrow 0}} O(\Delta t)+O(\Delta x)=0 .
$$

Portanto, o Método de Euler Implícito é consistente com a equação diferencial parcial. Pelos resultados obtidos em (2.3) o Método de Euler Implícito é convergente.

\subsection{Método de Crank-Nicolson}

O Método de Crank-Nicolson pode ser obtido tomando-se a média entre os métodos de Euler Explícito e Implícito. Assim, pelas expressões em (2.14) e (2.17), o Método de Crank-Nicolson é dado por:

$$
\begin{aligned}
\frac{U_{n}^{m+1}-U_{n}^{m}}{\Delta t} & =\frac{\mu_{n+\frac{1}{2}}\left(U_{n+1}^{m+1}+U_{n+1}^{m}-U_{n}^{m+1}-U_{n}^{m}\right)}{2 \Delta x^{2}}+\frac{f_{n}^{m+1}}{2} \\
& -\frac{\mu_{i-\frac{1}{2}}\left(U_{n}^{m+1}+U_{n}^{m}-U_{n-1}^{m}-U_{n-1}^{m+1}\right)}{2 \Delta x^{2}}+\frac{f_{n}^{m}}{2}
\end{aligned}
$$

$\operatorname{com} \mu_{n+\frac{1}{2}}=\mu\left(x_{n}+\frac{\Delta x}{2}\right), \operatorname{com} n=1,2, \ldots, N-1$ e $m=0,1, \ldots, M-1$, ou ainda,

$$
\begin{aligned}
& -\frac{\Delta t}{2 \Delta x^{2}} \mu_{n-\frac{1}{2}} U_{n-1}^{m+1}+\left(1+\frac{\Delta t}{2 \Delta x^{2}}\left(\mu_{n+\frac{1}{2}}+\mu_{n-\frac{1}{2}}\right)\right) U_{n}^{m+1}-\frac{\Delta t}{2 \Delta x^{2}} \mu_{n+\frac{1}{2}} U_{n+1}^{m+1}= \\
& \frac{\Delta t}{2 \Delta x^{2}} \mu_{n-\frac{1}{2}} U_{n-1}^{m}+\left(1-\frac{\Delta t}{2 \Delta x^{2}}\left(\mu_{n+\frac{1}{2}}-\mu_{n-\frac{1}{2}}\right)\right) U_{n}^{m}+\frac{\Delta t}{2 \Delta x^{2}} \mu_{n+\frac{1}{2}} U_{n+1}^{m}+\Delta t \bar{f}
\end{aligned}
$$

onde $\bar{f}=\frac{f_{n}^{m+1}+f_{n}^{m}}{2}$. 
Se $\mu(x)$ for um função constante constante então a expressão (2.22) pode ser escrita como:

$-\omega U_{n-1}^{m+1}+2(1+\omega) U_{n}^{m+1}-\omega U_{n+1}^{m+1}=\omega U_{n-1}^{m}+2(1-\omega) U_{n}^{m}+\omega U_{n+1}^{m}+\Delta t \bar{f}$,

$\operatorname{com} \omega=\mu \frac{\Delta t}{\Delta x^{2}}, n=1,2, \ldots, N-1$ e $m=0,1, \ldots, M-1$.

Note que, para obter a aproximação da solução no instante $t_{m+1}$, é necessária a resolução do seguinte sistema linear:

$$
A U_{m+1}=B U_{m}+C_{m+1}+\bar{F}_{m}, \quad \text { com } \quad m=0,1, \ldots, M-1 .
$$

onde,

$$
\begin{aligned}
& A=\left(\begin{array}{cccccc}
1+\omega & -\frac{\omega}{2} & 0 & \ldots & \ldots & 0 \\
0 & -\frac{\omega}{2} & 1+\omega & -\frac{\omega}{2} & \ldots & 0 \\
\vdots & & & & & \vdots \\
0 & \ldots & \ldots & 0 & -\frac{\omega}{2} & 1+\omega
\end{array}\right), \quad U_{m+1}=\left(\begin{array}{c}
U_{1}^{m+1} \\
U_{2}^{m+1} \\
\vdots \\
U_{N-2}^{m+1} \\
U_{N-1}^{m+1}
\end{array}\right) \\
& B=\left(\begin{array}{cccccc}
1-\omega & \frac{\omega}{2} & 0 & \ldots & \ldots & 0 \\
0 & \frac{\omega}{2} & 1-\omega & \frac{\omega}{2} & \ldots & 0 \\
\vdots & & & & & \vdots \\
0 & \cdots & \ldots & 0 & \frac{\omega}{2} & 1-\omega
\end{array}\right), \quad C_{m+1}=\frac{\omega}{2}\left(\begin{array}{c}
U_{0}^{m}+U_{0}^{m+1} \\
0 \\
\vdots \\
0 \\
U_{N}^{m}+U_{N}^{m+1}
\end{array}\right)
\end{aligned}
$$




$$
\text { e } \bar{F}_{m}=\frac{\Delta t}{2}\left(\begin{array}{c}
f_{1}^{m}+f_{1}^{m+1} \\
f_{1}^{m}+f_{1}^{m+1} \\
\vdots \\
f_{N-2}^{m}+f_{N-2}^{m+1} \\
f_{N-1}^{m}+f_{N-1}^{m+1}
\end{array}\right)
$$

O método descrito em (2.22) é chamado Método de Crank-Nicolson, e sua mólecula computacional é ilustrada na Figura (2.4).

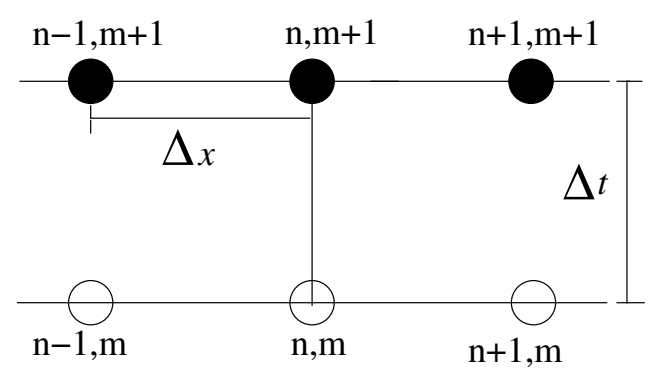

Figura 2.4: Molécula computacional do Método de Crank-Nicolson.

A Figura (2.4) ilustra a dependência que a aproximação obtida no ponto $\left(x_{n}, t_{m+1}\right)$ em relação aos pontos $\left(x_{n-1}, t_{m+1}\right),\left(x_{n+1}, t_{m+1}\right),\left(x_{n-1}, t_{m}\right),\left(x_{n}, t_{m}\right)$ e $\left(x_{n+1}, t_{m}\right)$, ou seja, é necessário conhecer-se os valores $U_{-1}^{m+1}, U_{n+1}^{m+1}, U_{n-1}^{m}$, $U_{n}^{m}$ e $U_{n+1}^{m}$ para que a aproximação $U_{n}^{m+1}$ possa ser calculada, a construção deste método numérico é discutida em [3], [4], [5] e [8].

\section{Condição de Estabilidade do Método de Crank-Nicolson}

De maneira análoga a feita aos outros métodos faz-se a substituição $u_{n}^{m}=$ $g^{m} e^{i n \theta}$ na expressão (2.23), adotando $\omega=\frac{\Delta t}{\Delta x^{2}} \mu_{M}$, onde $\mu_{M}=\max _{0 \leq x \leq L}\{\mu(x)\}$, assim obtém-se: 


$$
\begin{aligned}
& -\frac{\Delta t}{\Delta x^{2}} \mu_{M} g^{m+1} e^{i(n-1) \theta}+2\left(1+\frac{\Delta t}{\Delta x^{2}} \mu_{M}\right) g^{m+1} e^{i n \theta}-\frac{\Delta t}{\Delta x^{2}} \mu_{M} g^{m+1} e^{i(n+1) \theta}= \\
& =\frac{\Delta t}{\Delta x^{2}} \mu_{M} g^{m} e^{i(n-1) \theta}+2\left(1-\frac{\Delta t}{\Delta x^{2}} \mu_{M}\right) g^{m} e^{i n \theta}+\frac{\Delta t}{\Delta x^{2}} \mu_{M} g^{m} e^{i(n+1) \theta} .
\end{aligned}
$$

Agrupando os termos semelhantes,

$$
\begin{aligned}
& -\frac{\Delta t}{\Delta x^{2}} \mu_{M} g g^{m} e^{i n \theta} e^{-\theta}+2\left(1+\frac{\Delta t}{\Delta x^{2}} \mu_{M}\right) g g^{m} e^{i n \theta}-\frac{\Delta t}{\Delta x^{2}} \mu_{M} g g^{m} e^{i n \theta} e^{i \theta}= \\
& =\frac{\Delta t}{\Delta x^{2}} \mu_{M} g^{m} e^{i n \theta} e^{-i \theta}+2\left(1-\frac{\Delta t}{\Delta x^{2}} \mu_{M}\right) g^{m} e^{i n \theta}+\frac{\Delta t}{\Delta x^{2}} \mu_{M} g^{m} e^{i n \theta} e^{i \theta} .
\end{aligned}
$$

Logo,

$-\frac{\Delta t}{\Delta x^{2}} \mu_{M} g\left(e^{-i \theta}+g e^{i \theta}\right)+2\left(1+\frac{\Delta t}{\Delta x^{2}} \mu_{M}\right) g=\frac{\Delta t}{\Delta x^{2}} \mu_{M}\left(e^{-i \theta}+e^{i \theta}\right)+2\left(1-\frac{\Delta t}{\Delta x^{2}} \mu_{M}\right)$

Lembrando que $e^{a+b i}=e^{a}(\cos (b)+i \sin (b))$, tem-se

$$
\begin{aligned}
-2 \frac{\Delta t}{\Delta x^{2}} \mu_{M} g \cos (\theta)+2\left(1+\frac{\Delta t}{\Delta x^{2}} \mu_{M}\right) g & =2 \frac{\Delta t}{\Delta x^{2}} \mu_{M} \cos (\theta)+2\left(1-\frac{\Delta t}{\Delta x^{2}} \mu_{M}\right) \Rightarrow \\
g\left(1+\frac{\Delta t}{\Delta x^{2}} \mu_{M}(1-\cos (\theta))\right) & =\frac{\Delta t}{\Delta x^{2}} \mu_{M}(\cos (\theta)-1)+1
\end{aligned}
$$

e utilizando a relação trigonométrica $\cos (x)-1=-2 \sin ^{2}\left(\frac{x}{2}\right)$, tem-se

$$
g\left(1+2 \frac{\Delta t}{\Delta x^{2}} \mu_{M} \sin ^{2}\left(\frac{\theta}{2}\right)\right)=1-2 \frac{\Delta t}{\Delta x^{2}} \mu_{M} \sin ^{2}\left(\frac{\theta}{2}\right)
$$

$\log 0$

$$
g=\frac{1-2 \frac{\Delta t}{\Delta x^{2}} \mu_{M} \sin ^{2}\left(\frac{\theta}{2}\right)}{1+2 \frac{\Delta t}{\Delta x^{2}} \mu_{M} \sin ^{2}\left(\frac{\theta}{2}\right)}<1, \quad \forall \quad \theta .
$$

Portanto, o Método de Crank-Nicolson é incondicionalmente estável. 


\section{Consistência do Método de Crank-Nicolson}

Suponha que $u(x, t)$ seja uma função suficientemente suave, $P u(x, t)$ a equação diferencial e $P_{\Delta} u$ o método de Crank-Nicolson, com $u_{n}^{m}=u\left(x_{n}, t_{m}\right)$ e $\mu_{M}=\max _{0 \leq x \leq L}\{\mu(x)\}$, então

$$
P_{\Delta} u=\frac{u_{n}^{m+1}-u_{n}^{m}}{\Delta t}-\frac{\mu_{M}}{2 \Delta x^{2}}\left(u_{n-1}^{m}+u_{n+1}^{m}-2\left(u_{n}^{m}+u_{n}^{m+1}\right)+u_{n-1}^{m+1}+u_{n+1}^{m+1}\right),
$$

Note que, aplicando as expansões de (2.5), obtém-se

- $\frac{u_{n}^{m+1}-u_{n}^{m}}{\Delta t}=u_{t}+O(\Delta t)$

- $\frac{u_{n-1}^{m}-2 u_{n}^{m}+u_{n+1}^{m}}{\Delta x^{2}}=u_{x x}+O\left(\Delta x^{2}\right)$,

- $\frac{u_{n-1}^{m}-2 u_{n}^{m}+u_{n+1}^{m}}{\Delta x^{2}}=u_{x x}^{+}+O\left(\Delta x^{2}\right)$,

onde todas as derivadas são calculadas no ponto $\left(x_{n}, t_{m}\right)$, única exceção feita a $u_{x x}^{+}$que é calculada no ponto $\left(x_{n}, t_{m}+\Delta t\right)$.

Substituindo as aproximações na equação (2.24) segue que

$$
P_{\Delta} u=u_{x}+O(\Delta t)-\mu_{M} \frac{u_{x x}+u_{x x}^{+}+O\left(\Delta x^{2}\right)}{2} .
$$

Logo,

$$
\begin{aligned}
\lim _{\substack{\Delta t \rightarrow 0 \\
\Delta x \rightarrow 0}} P u-P_{\Delta} u & =\lim _{\substack{\Delta t \rightarrow 0 \\
\Delta x \rightarrow 0}}\left(u_{t}-\mu_{M} u_{x x}-u_{t}+O(\Delta t)+\mu_{M} \frac{u_{x x}+u_{x x}^{+}+O\left(\Delta x^{2}\right)}{2}\right) \Longrightarrow \\
\lim _{\substack{\Delta t \rightarrow 0 \\
\Delta x \rightarrow 0}} P u-P_{\Delta} u & =u_{t}-\mu_{M} u_{x x}-u_{t}+\mu_{M} \frac{u_{x x}+u_{x x}}{2}=0 .
\end{aligned}
$$

Portanto, o Método de Crank-Nicolson é consistente com a equação diferencial. Pelos resultados obtidos em (2.4), aplicando o Teorema de Lax Richtmyer o método de Crank-Nicolson é convergente. 


\section{Capítulo 3}

\section{A Técnica do Super-Passo}

A Técnica do Super-Passo é uma forma simples e eficiente de se reciclar códigos explícitos, melhorando a sua performace computacional. A Técnica do Super-Passo é baseada nos Métodos Runge-Kutta-Chebyshev, como pode ser visto em [28]. Assim como os Métodos Runge-Kutta, a Técnica do SuperPasso é composta por $N$ sub-passos (estágios) e relaxa a restrição temporal imposta pela condição de estabilidade do método numérico explícito.

Neste Capítulo, a fim de tornar a compreensão da técnica a mais simples possível, é apresentada a formulação da Técnica do Super-Passo aplicada ao Método de Euler Explícito para um sistema de equações diferenciais ordinárias. O método aqui descrito poder ser aplicado em malhas estruturadas e não estruturadas na resolução numérica de equações diferenciais parciais do tipo parabólico lineares e não lineares para uma, duas ou três dimensões. É possível resolver numericamente sistemas de equações para escoamentos de fluídos multi-fásicos, ou ainda, equações de advecção-difusão. Os detalhes são discutidos em [2], [18], [21], [29], [13]. 
Considere o sistema de equações diferenciais ordinárias

$$
\left\{\begin{array}{l}
\frac{d U}{d t}+A U(t)=0, \quad t>0 \\
U(0)=U_{0}
\end{array}\right.
$$

onde $A$ é uma matriz $n \times n$ simétrica definida positiva e $U_{0}$ é um vetor em $\Re^{n}$ dado.

O Método de Euler Explícito para o sistema (3.1) pode ser escrito como

$$
\left\{\begin{array}{l}
U^{m+1}=(I-\Delta t A) U^{m}, \quad m=0,1, \ldots \\
U^{0}=U_{0}
\end{array}\right.
$$

sendo $\Delta t>0$ o passo de integração no tempo, $I$ a matriz identidade de ordem $n$ e $U^{m} \in \Re^{n}$ um vetor que representa a aproximação obtida pelo método numérico (3.2) para a solução do sistema (3.1) no instante de tempo $t_{m}=m \Delta t$.

O esquema numérico em (3.2) é estável se

$$
\rho(I-\Delta t A)<1
$$

sendo que $\rho(\cdot)$ denota o raio espectral da matriz $(I-\Delta t A), \lambda_{\max }$ o seu maior autovalor e $\lambda_{\min }$ o menor. A condição em (3.3) é satisfeita com a escolha

$$
\Delta t<\Delta t_{\text {expl }}=\frac{2}{\lambda_{\max }}
$$

A Técnica do Super-Passo procura relaxar a restrição ao passo de integração no tempo imposta por (3.4); assim, em analogia aos Método RungeKutta de $N$ estágios, a condição de estabilidade não é atendida ao final de todo o passo de integração $\Delta t$, mas apenas ao final de um ciclo de $N$ passos no tempo. 
Um passo de integração no tempo da Técnica do Super-Passo $\Delta T$, consiste de $N$ sub-passos (estágios) $\tau_{i}$, com $i=1, \ldots, N$, ou seja, para cada super-passo de integração no tempo $\Delta T$ são calculados os $N$ sub-passos.

$$
\begin{gathered}
U^{m+1,1}=\left(I-A \tau_{1}\right) U^{m}, \\
U^{m+1,2}=\left(I-A \tau_{2}\right) U^{m+1,1}, \\
\vdots \\
U^{m+1}=U^{m+1, N}=\left(I-A \tau_{N}\right) U^{m+1, N-1} .
\end{gathered}
$$

Substituindo recursivamente até um ciclo $j$ obtém-se uma expressão para os primeiros $j$ sub-passos,

$$
U^{m+1, j}=\prod_{i=1}^{j}\left(I-A \tau_{i}\right) U^{m}, \quad j=1,2, \ldots, N \quad m=0,1 \ldots
$$

A demonstração desta igualdade segue por indução sobre $j$.

A Técnica do Super-Passo aplicada ao Método de Euler Explícito com $N$ sub-passos é dada por

$$
U^{m+1}=\prod_{i=1}^{N}\left(I-A \tau_{i}\right) U^{m}, \quad m=0,1, \ldots
$$

onde $A$ é uma matriz $n \times n$ simétrica definida positiva, $I$ é a matriz identidade de ordem $n, N$ a quantidade de sub-passos, $\tau_{i}$ o i-ésimo sub-passo, que será explicitado na próxima Seção, e $U^{m}$ representa aproximação numérica para a solução do sistema em (3.1) no instante de tempo $t_{m}$. 


\subsection{Condição de Estabilidade}

A Técnica do Super-Passo procura relaxar a restrição ao passo de integração no tempo imposta por (3.4), assim a condição de estabilidade não é atendida ao final de todo o passo de integração $\Delta t$, mas apenas ao final de um ciclo de $N$ passos no tempo. A condição de estabilidade é imposta sobre o super-passo $\Delta T$, enquanto tenta-se maximizar a soma $\Delta T=\sum_{i=1}^{N} \tau_{i}$.

Uma condição suficiente para que a condição de estabilidade do algoritmo (3.5) dada em (3.3) seja atendida é,

$$
\prod_{i=1}^{N}\left|1-\lambda \tau_{i}\right|<1, \quad \forall \lambda \in\left[\lambda_{\min }, \lambda_{\max }\right]
$$

onde $\prod_{i=1}^{N}\left|1-\lambda \tau_{i}\right|$ é o fator de amplificação do método e $\lambda_{\min }$ e $\lambda_{\max }$ são o menor e maior autovalor da matriz $A$.

Existem vários valores de $\tau_{i}$ que satisfazem a condição acima, mas o objetivo é determinar $\tau^{\prime} s$ tais que:

$$
\Delta T=\sum_{i=1}^{N} \tau_{i} \quad \text { seja o maior possível. }
$$

Entretanto, o problema em (3.6) não é conveniente, pois a condição de estabilidade possui uma desigualdade estrita. A condição (3.6) será substituída por

$$
\prod_{i=1}^{N}\left|1-\lambda \tau_{i}\right| \leq k, \quad \forall \lambda \in\left[\epsilon, \lambda_{\max }\right],
$$

onde $\epsilon \in\left(0, \lambda_{\min }\right]$ e $k \in(0,1)$, ambos fixos.

Note que $k$ pode ser escolhido arbitrariamente próximo de 1 , bastando escolher $\epsilon$ suficientemente próximo de $\lambda_{\min }$. 
O problema de encontrar os valores "ótimos" para os $\tau^{\prime} s$ pode ser reescrito como encontrar $\tau_{1}, \tau_{2}, \ldots, \tau_{N}$ tais que

$$
P_{N}(\lambda)=\prod_{i=1}^{N}\left(1-\lambda \tau_{i}\right)
$$

satisfaça

$$
\begin{gathered}
\left|P_{N}(\lambda)\right| \leq k, \quad k<1 \quad \forall \lambda \in\left[\epsilon, \lambda_{\max }\right] \quad \text { (estabilidade) } \\
\left|\frac{d P_{N}}{d \lambda}(0)\right|=\sum_{i=1}^{N} \tau_{i}, \quad \text { (valores ótimos). }
\end{gathered}
$$

Considere o seguinte polinômio de grau $N$ em $\lambda$,

$$
Q_{N}(\lambda)=\frac{T_{N}\left(\frac{\lambda_{\max }+\epsilon-2 \lambda}{\lambda_{\max }-\epsilon}\right)}{T_{N}\left(\frac{\lambda_{\max }+\epsilon}{\lambda_{\max }-\epsilon}\right)},
$$

onde $\epsilon \in\left(0, \lambda_{\min }\right]$ e $\lambda \in\left[\epsilon, \lambda_{\max }\right]$.

O numerador é um Polinômio de Chebyshev de grau $N$ definido no intervalo $\left[\epsilon, \lambda_{\max }\right]$, e o denominador é uma constante.

Pelas propriedades dos Polinômios de Chebyshev, tem-se que,

$$
\left|T_{N}\left(\frac{\lambda_{\max }+\epsilon-2 \lambda}{\lambda_{\max }-\epsilon}\right)\right| \leq 1
$$

Assim, o polinômio em (3.9) é limitado por

$$
k^{\prime}=\frac{1}{T_{N}\left(\frac{\lambda_{\max }+\epsilon}{\lambda_{\max }-\epsilon}\right)},
$$


note que

$$
\frac{\lambda_{\max }+\epsilon}{\lambda_{\max }-\epsilon}>1
$$

e, pelas propriedades dos polinômios de Chebyshev, tem-se que

$$
\left|T_{N}\left(\frac{\lambda_{\max }+\epsilon}{\lambda_{\max }-\epsilon}\right)\right|>1
$$

onde $\epsilon \in\left(0, \lambda_{\min }\right]$ e $0<\lambda_{\max }$.

Então

$$
\left|k^{\prime}\right|<1
$$

As raízes do polinômio $Q_{N}(\lambda)$ são as mesmas do Polinômio de Chebyshev $T_{N}\left(\frac{\lambda_{\max }+\epsilon-2 \lambda}{\lambda_{\max }-\epsilon}\right)$ e são dadas por:

$$
\lambda_{i}=\frac{1}{2}\left(\left(-\lambda_{\max }+\epsilon\right) \cos \left(\frac{2 i-1}{N} \frac{\pi}{2}\right)+\lambda_{\max }+\epsilon\right), \quad i=1, \ldots, N
$$

onde $\epsilon \in\left(0, \lambda_{\min }\right)$.

Voltando ao fator de amplificação da Técnica do Super-Passo, que é dado pela função,

$$
P_{N}(\lambda)=\prod_{i=1}^{N}\left(1-\lambda \tau_{i}\right) .
$$

Procura-se determinar quais valores de $\tau^{\prime} s$ satisfazem:

$$
\begin{gathered}
\left|P_{N}(\lambda)\right| \leq k, \quad \forall \lambda \in\left[\epsilon, \lambda_{\max }\right], \quad k<1 \\
\left|P_{N}^{\prime}(0)\right|=\sum_{i=1}^{N} \tau_{i} .
\end{gathered}
$$

Escolhe-se

$$
\tau_{i}=\frac{1}{\lambda_{i}}, \quad \text { com } \quad i=1, \ldots N
$$


Então, (3.10) pode ser escrito como,

$$
P_{N}(\lambda)=\prod_{i=1}^{N}\left(1-\lambda \frac{1}{\lambda_{i}}\right), \quad \text { com } \quad i=1, \ldots N
$$

Assim, as $N$ raízes de (3.10) são dadas por $\lambda_{i}, i=1, \ldots, N$.

Logo, têm-se dois polinômios, $P_{N}$ e $Q_{N}$ de grau $N$ com as mesmas $N$ raízes $\lambda_{i}$, e $P_{N}(0)=Q_{N}(0)=1$.

Portanto,

$$
P_{N}(\lambda) \equiv Q_{N}(\lambda), \quad \forall \lambda \in\left(\epsilon, \lambda_{\max }\right]
$$

A condição em (3.11) é garantida escolhendo $k=k^{\prime}$, ou seja,

$$
\left|P_{N}(\lambda)\right| \equiv\left|Q_{N}(\lambda)\right| \leq\left|k^{\prime}\right|<1, \quad \forall \lambda \in\left(\epsilon, \lambda_{\max }\right] .
$$

Pelos artigos [2], [1] e [18], sabe-se que o maior valor para a soma em (3.12) é obtido escolhendo,

$$
\tau_{i}=\frac{1}{\lambda_{i}}, \quad \text { com } \quad i=1, \ldots, N
$$

Os sub-passos de integração no tempo $\tau^{\prime} s$ são dados explicitamente por,

$$
\tau_{i}=2\left(\left(-\lambda_{\max }+\epsilon\right) \cos \left(\frac{2 i-1}{N} \frac{\pi}{2}\right)+\lambda_{\max }+\epsilon\right)^{-1}, \quad i=1, \ldots, N
$$

que podem ser reescritos mais convenientemente como,

$$
\tau_{i}=\Delta t_{\text {expl }}\left((-1+\nu) \cos \left(\frac{2 i-1}{N} \frac{\pi}{2}\right)+1+\nu\right)^{-1}, \quad i=1, \ldots, N
$$

onde $\nu=\frac{\epsilon}{\lambda_{\max }}, \quad 0<\nu<1, \Delta t_{\text {expl }}=\frac{2}{\lambda_{\max }}$ e $N$ é a quantidade de sub-passos. 
Note que se $N=1$ e $\nu=0$ segue que $\tau_{1}=\Delta t_{\text {expl }}$, ou seja, a Técnica do Super-Passo realiza as mesmas aproximações do Método de Euler Explícito.

Utilizando a condição (3.12) e uma das formulações dos Polinômios de Chebyshev o tamanho do super-passo, $\Delta T$, pode ser expresso explicitamente como,

$$
\Delta T=\sum_{i=1}^{N} \tau_{i}=\Delta t_{e x p l} \frac{N}{2 \sqrt{\nu}}\left(\frac{(1+\sqrt{\nu})^{2 N}-(1-\sqrt{\nu})^{2 N}}{(1+\sqrt{\nu})^{2 N}+(1-\sqrt{\nu})^{2 N}}\right)
$$

Assim,

$$
\Delta T \underset{\nu \rightarrow 0}{\longrightarrow} N^{2} \Delta t_{e x p l}
$$

isso é,

$$
\frac{\Delta T}{\Delta t_{\text {expl }}} \underset{\nu \rightarrow 0}{\longrightarrow} N^{2}
$$

A Tabela (3.1) apresenta os diferentes valores do super-passo de integração no tempo quando $\nu \rightarrow 0$, e a aproximação numérica para $N^{2}$ no limite (3.15). Para se obter os resultados tabelados aplicou-se a (3.14) com 9 sub-passos, e o passo de integração no tempo, determinado pela condição de estabilidade do Método de Euler Explícito é $\Delta t_{\text {expl }}=1.851852 \times 10^{-6}$.

\begin{tabular}{|c|c|c|}
\hline$\nu$ & $\Delta T$ & $N^{2}$ \\
\hline \hline $10^{-1}$ & $2.635191 \times 10^{-5}$ & 14.23 \\
\hline $10^{-2}$ & $7.895230 \times 10^{-5}$ & 42.63 \\
\hline $10^{-3}$ & $1.356929 \times 10^{-4}$ & 73.27 \\
\hline $10^{-4}$ & $1.484056 \times 10^{-4}$ & 80.13 \\
\hline $10^{-5}$ & $1.498387 \times 10^{-4}$ & 80.91 \\
\hline $10^{-6}$ & $1.499839 \times 10^{-4}$ & 80.99 \\
\hline
\end{tabular}

Tabela 3.1: Aproximação numérica para o limite (3.15).

Os resultados na Tabela (3.1) mostram que a aproximação numérica para $N^{2}$ tende a 81 quando $\nu \rightarrow 0$. 
Note que $N$ passos de um método explícito de comprimento $\Delta t_{\text {expl }}$ calcula aproximações em um instante final $N \Delta t_{\text {expl }}$, mas a Técnica do Super-Passo com $N$ sub-passos realiza aproximações para um instante de tempo $t=$ $N^{2} \Delta t_{\text {expl }}$ quando $\nu \rightarrow 0$.

Assim a Técnica do Super-Passo cobre um intervalo $N$ vezes maior com custo computacional adicional de calcular (3.13) no primeiro ciclo dos $N$ sub-passos no tempo.

\subsection{Consistência}

A Técnica do Super-Passo será consistente se o método explícito a que ela for aplicada o for, pois a técnica altera apenas o tamanho do passo no tempo.

\subsection{Erro Cometido na Aproximação pela Técnica do Super-Passo}

Para analisar o erro cometido entre a aproximação gerada pela Técnica do Super-Passo e a solução analítica de um problema, para manter a simplicidade e clareza dos resultados obtidos considerou-se o problema modelo proposto em (3.1) com $n=1$, ou seja, considerou-se o caso em que o sistema de equações diferenciais é unidimensional. Assim o problema (3.1) possui como solução $u(t)=u_{0} e^{-\lambda t}$, o erro cometido na aproximação pela Técnica do Super-Passo aplicada ao Método de Euler Explícito após $k$ passos no 
tempo é dado por:

$$
\begin{aligned}
\left|e^{(k)}\right| & \equiv\left|u(k \Delta T)-U^{(k)}\right|=\left|e^{-\lambda k \Delta T}-\prod_{i=1}^{N}\left(1-\lambda \tau_{i}\right) U^{(k-1)}\right|= \\
& =\left|e^{-\lambda \Delta T} e^{-\lambda(k-1) \Delta T}-\prod_{i=1}^{N}\left(1-\lambda \tau_{i}\right) U^{(k-1)}\right|= \\
& =\left|e^{-\lambda \Delta T} u((k-1) \Delta T)-\prod_{i=1}^{N}\left(1-\lambda \tau_{i}\right) U^{(k-1)}\right| .
\end{aligned}
$$

Somando e subtraindo $\prod_{i=1}^{N}\left(1-\lambda \tau_{i}\right) u((k-1) \Delta T)$ segue

$$
\begin{aligned}
\left|e^{k}\right| & \equiv \mid e^{-\lambda \Delta T} u((k-1) \Delta T)-\prod_{i=1}^{N}\left(1-\lambda \tau_{i}\right) u((k-1) \Delta T)+ \\
& +\prod_{i=1}^{N}\left(1-\lambda \tau_{i}\right) u((k-1) \Delta T)-\prod_{i=1}^{N}\left(1-\lambda \tau_{i}\right) U^{(k-1)} \mid= \\
& =\mid\left[e^{-\lambda \Delta T}-\prod_{i=1}^{N}\left(1-\lambda \tau_{i}\right)\right] u((k-1) \Delta T)+ \\
& +\left[u((k-1) \Delta T)-U^{(k-1)}\right] \prod_{i=1}^{N}\left(1-\lambda \tau_{i}\right) \mid \leq \\
& \leq\left|e^{-\lambda \Delta T}-\prod_{i=1}^{N}\left(1-\lambda \tau_{i}\right)\right||u((k-1) \Delta T)|+ \\
& +\left|u((k-1) \Delta T)-U^{(k-1)}\right|\left|\prod_{i=1}^{N}\left(1-\lambda \tau_{i}\right)\right|
\end{aligned}
$$


Portanto,

$$
\left|e^{(k)}\right| \leq\left|e^{-\lambda \Delta T}-\prod_{i=1}^{N}\left(1-\lambda \tau_{i}\right)\right||u((k-1) \Delta T)|+\left|u((k-1) \Delta T)-U^{(k-1)}\right|\left|\prod_{i=1}^{N}\left(1-\lambda \tau_{i}\right)\right|
$$

Observe que,

- $|u((k-1) \Delta T)|=\left|u_{0} e^{-\lambda}(k-1) \Delta T\right| \leq\left|u_{0}\right|$,

- $\left|\prod_{i=1}^{N}\left(1-\lambda \tau_{i}\right)\right|<1$, condição de estabilidade.

Substituindo em (3.16), tem-se

$$
\left|e^{k}\right| \leq\left|e^{-\lambda \Delta T}-\prod_{i=1}^{N}\left(1-\lambda \tau_{i}\right)\right|\left|u_{0}\right|+\left|e^{k-1}\right| .
$$

Note que o erro cometido no instante de tempo $t_{k}$ pela Técnica do SuperPasso é dado em função do erro cometido no instante de tempo anterior. Logo,

$$
\left|e^{k-1}\right| \leq\left|e^{-\lambda \Delta T}-\prod_{i=1}^{N}\left(1-\lambda \tau_{i}\right)\right|\left|u_{0}\right|+\left|e^{k-2}\right|
$$

Aplicando recursivamente até o termo $\left|e^{0}\right|=0$ obtém-se

$$
\left|e^{k}\right| \leq k\left|e^{-\lambda \Delta T}-\prod_{i=1}^{N}\left(1-\lambda \tau_{i}\right)\right|\left|u_{0}\right|
$$

A expansão em série de Taylor $e^{-\lambda \Delta T}$ é dada por:

$$
e^{-\lambda \Delta T}=1-\lambda \Delta T+\frac{(\lambda \Delta T)^{2}}{2}-\frac{(\lambda \Delta T)^{3}}{6}+\frac{(\lambda \Delta T)^{4}}{24}+O\left(\Delta T^{5}\right) .
$$

Substituindo em (3.17) e mantendo apenas os termos de ordem mais baixa tem-se: 


$$
\left|e^{k}\right| \leq k \frac{\lambda_{\max }}{2}\left(\sum_{i=1}^{N} \tau_{i=1}^{2}\right)\left|u_{0}\right|
$$

onde $\lambda_{\max }=\frac{2}{\Delta t_{\operatorname{expl}}}$.

A expressão (3.18) depende apenas de $\Delta t_{\text {expl }}$, assim o método é de ordem 1 no tempo. Note que pela equação (3.13) quanto maior for $\nu$ menor será o comprimento de cada $\tau$ e consequentemente pela expressão (3.18) o erro também será menor. 


\section{Capítulo 4}

\section{Resultados Numéricos}

O presente Capítulo apresenta as validações por refinamento de malha e a comparação de performance dos métodos de Euler Explícito, Euler Implícito e Crank-Nicolson com o método resultante da conjunção da Técnica do SuperPasso ao Método de Euler Explícito para a aproximação numérica da solução da equação do calor.

Os métodos foram implementados em linguagem de programação FORTRAN 90 utilizando precisão dupla. Para medir o tempo de processamento dos métodos numéricos utilizados foi utilizada a subrotina implícita da linguagem FORTRAN 90 timef. (maiores detalhes sobre a linguagem FORTRAN podem ser encontradas em [6]).

\subsection{Análise Numérica de Convergência}

Antes de analisar a convergência dos métodos numéricos é necessário descrever qual será a norma adotada e como a análise de convergência numérica será realizada.

Seja $\vec{e}$ um vetor real de dimensão $n$ arbitrário. Então a norma infinito 
deste vetor será dada por

$$
\|e\|_{\infty}=\max _{0 \leq i \leq n}\left\{\left|e_{i}\right|\right\}
$$

Considere a seguinte equação diferencial parcial do tipo parabólico juntamente com as suas condições de contorno e inicial

$$
\left\{\begin{array}{l}
u_{t}(x, t)-\left[\mu(x) u_{x}(x, t)\right]_{x}=f(x, t), \\
u(x, 0)=\phi(x) \quad x \in[0, L] \\
u(0, t)=g(t) \quad t \in[0, T] \\
u(L, t)=h(t) \quad t \in[0, T]
\end{array}\right.
$$

definida no domínio $\Omega=[0, L] \times[0, T], \operatorname{com} \mu(x)>0, \forall x \in[0, L]$.

Dividiu-se o intervalo $[0, L]$, da variável espacial $x$, em $N$ partes iguais de comprimento $\Delta x$, obtendo os $N+1$ pontos $x_{i}=i \Delta x, i=0,1, \ldots, N$, onde $\Delta x=\frac{L}{N}$, particionando o intervalo $[0, T]$ da variável temporal $t$ pelo passo de integração no tempo $\Delta t$, que é determinado respeitando a condição de estabilidade do método numérico, obtém-se a quantidade de passos no tempo $M$, ou seja, tem-se $M+1$ pontos $t_{i}=i \Delta t, i=0,1, \ldots, M$.

A solução $u(x, t)$ do problema (4.2) nos pontos do domínio discretizado $\Omega_{\Delta x}^{\Delta t}=\left\{\left(x_{i}, t_{j}\right) \in \Omega / x_{i}=i \Delta x \quad\right.$ e $\left.t_{j}=j \Delta t\right\}$ é representada por $u\left(x_{i}, t_{j}\right)=$ $u_{i, j}$ e a aproximação numérica obtida por algum método numérico será denotada por $U_{i, j}$.

A aproximação obtida por um método numérico para aproximar a solução do problema proposto em (4.2), possui um erro que é dado por $e_{i, j}=\mid u_{i, j}$ $U_{i, j} \mid, \operatorname{com} i=0,1, \ldots, N$ e $j=0,1, \ldots, M$.

Para realizar a análise de convergência assumir-se-á que a solução numérica obtida pelo método numérico possui uma expansão assintótica em potências do passo de integração no espaço. Assim, supondo que $u(x, t)$ possui uma solução numérica da forma $U(x, t, \Delta x)$, para $\Delta x$ suficientemente pequeno 
pode se escrever a expansão assintótica.

$$
U(x, t, \Delta x)=u(x, t)+E_{q}(x, t) \Delta x^{q}+E_{q+1}(x, t) \Delta x^{q+1}+\ldots,
$$

onde $u(x, t)$ é a solução exata, e com coeficientes funcionais $E_{i}(x, t), i=$ $q, q+1, \ldots$ independem de $\Delta x$.

No problema apresentado na validação e na comparação de performance dos métodos numéricos, a solução analítica $u(x, t)$ é conhecida a priori. Para este problema, as expansões de $U(x, t, \Delta x)$ e $U\left(x, t, \frac{\Delta x}{2}\right)$, para o mesmo ponto $(x, t)$, podem ser expressas como

$$
\begin{aligned}
& U(x, t, \Delta t) \approx u(x, t)+E_{q}(x, t) \Delta t^{q}, \\
& U\left(x, t, \frac{\Delta t}{2}\right) \approx u(x, t)+E_{q}(x, t) \frac{\Delta t^{q}}{2^{q}} .
\end{aligned}
$$

Tais aproximações permitem estimar a razão de convergência do método numérico

$$
\frac{|U(x, t, \Delta t)-u(x, t)|_{\infty}}{\left|U\left(x, t, \frac{\Delta t}{2}\right)-u(x, t)\right|_{\infty}} \approx 2^{q} .
$$

A convergência da razão em (4.5) para $2,4,8, \ldots$, significa que o método numérico tem ordem $q=1,2,3, \ldots$, respectivamente.

A estimativa da razão de convergência em (4.5) será utilizada na validação dos métodos numéricos implementados.

A implementação dos métodos numéricos foi validada empregando a análise de convergência explicada anteriormente, utilizando o seguinte problema modelo:

$$
\left\{\begin{array}{l}
u_{t}-\left[\mu(x) u_{x}\right]_{x}=f(x, t), \\
u(x, 0)=\cos (\pi x), \quad 0 \leq x \leq \frac{1}{4} ; \quad 0 \leq t \leq \frac{1}{4}, \\
u(0, t)=\cos (\pi t), \quad u\left(\frac{1}{4}, t\right)=\frac{\sqrt{2}}{2} \cos (\pi t), \\
\mu_{x}(x)=\frac{1}{2}, \quad \mu(x)=\frac{x}{2}+1,
\end{array}\right.
$$


onde $f(x, t)=u_{t}-\left[\mu(x) u_{x}\right]_{x}$ e $u(x, t)=\cos (\pi x) \cos (\pi t)$.

\subsubsection{Método de Euler Explícito}

O passo no tempo para o método de Euler Explícito foi $\Delta t=\frac{\Delta x^{2}}{3}$, note que esta escolha respeita a condição de estabilidade do método, como visto em (2.2.1). Definiu-se $\Delta t_{\text {expl }}=\frac{\Delta x^{2}}{3}$ como sendo delta $t$ explícito.

As tabelas a seguir apresentam, $N_{x}, N_{T}$ as respectivas divisões do intervalo de definição nas direções $x$ e $t$, o símbolo $\Delta x$ representa o passo de integração no espaço, $\|e\|_{\infty}$ o erro máximo cometido na aproximação e razão a ordem do método numérico ao quadrado.

\begin{tabular}{|c|c|c|c|c|}
\hline$N_{x}$ & $\Delta x$ & $N_{T}$ & $\|e\|_{\infty}$ & Razão \\
\hline \hline 25 & $1.00 \times 10^{-2}$ & 8438 & $5.387633 \times 10^{-6}$ & \\
\hline 50 & $5.00 \times 10^{-3}$ & 33751 & $1.347009 \times 10^{-6}$ & 3.999700 \\
\hline 100 & $2.50 \times 10^{-3}$ & 135000 & $3.368690 \times 10^{-7}$ & 3.998613 \\
\hline 200 & $1.25 \times 10^{-3}$ & 540000 & $8.421701 \times 10^{-8}$ & 4.000011 \\
\hline 400 & $6.25 \times 10^{-4}$ & 2160000 & $2.105431 \times 10^{-8}$ & 3.999989 \\
\hline
\end{tabular}

Tabela 4.1: Resultados numéricos obtidos pelo Método de Euler Explícito na aproximação da solução do problema (4.6).

A tabela acima mostra que quando o passo de integração no espaço, $\Delta x$, é dividido por dois o erro cometido na aproximação diminui e a razão entre os erros tende a 4 .

Assim, como foi utilizado $\Delta t_{\text {expl }}=\frac{\Delta x^{2}}{3 \mu_{\max }}$, onde $\mu_{\max }=\mu(1)=1.5$, conclui-se que o Método de Euler Explícito tem ordem 1.

\subsubsection{Método de Euler Implícito}

A análise de convergência numérica para o Método de Euler Implícito foi realizada adotando-se $\Delta t=\Delta x$. Os resultados obtidos encontram-se na 
Tabela 4.2, abaixo:

\begin{tabular}{|c|c|c|c|}
\hline$N_{x}=N_{T}$ & $\Delta x=\Delta t$ & $\|e\|_{\infty}$ & Razão \\
\hline \hline 25 & $1.00 \times 10^{-2}$ & $2.369216 \times 10^{-4}$ & \\
\hline 50 & $5.00 \times 10^{-3}$ & $1.190450 \times 10^{-4}$ & 1.990185 \\
\hline 100 & $2.50 \times 10^{-3}$ & $5.966869 \times 10^{-5}$ & 1.995099 \\
\hline 200 & $1.25 \times 10^{-3}$ & $2.987136 \times 10^{-5}$ & 1.997521 \\
\hline 400 & $6.25 \times 10^{-4}$ & $1.494509 \times 10^{-5}$ & 1.998740 \\
\hline
\end{tabular}

Tabela 4.2: Resultados numéricos obtidos pelo Método de Euler Implícito na aproximação da solução do problema (4.6)

Como se nota, as razões de convergência (coluna 5 da Tabela (4.2)) tendem a 2 quando os passos de integração tendem a zero, donde conclui-se que o Método de Euler Implícito é de primeira ordem (note que, desta vez, $\Delta t=\Delta x)$.

\subsubsection{Método de Crank-Nicolson}

Para validar a implementação do Método de Crank-Nicolson escolheu-se $\Delta t=\Delta x$ e refinou-se progressivamente a malha empregando 2 como razão de refinamento. Os resultados encontram-se na Tabela (4.3) 


\begin{tabular}{|c|c|c|c|}
\hline$N_{x}=N_{T}$ & $\Delta x=\Delta t$ & $\|e\|_{\infty}$ & Razão \\
\hline \hline 25 & $1.00 \times 10^{-2}$ & $1.071806 \times 10^{-5}$ & \\
\hline 50 & $5.00 \times 10^{-3}$ & $2.678459 \times 10^{-6}$ & 4.001577 \\
\hline 100 & $2.50 \times 10^{-3}$ & $6.698336 \times 10^{-7}$ & 3.998693 \\
\hline 200 & $1.25 \times 10^{-3}$ & $1.674572 \times 10^{-7}$ & 4.000028 \\
\hline 400 & $6.25 \times 10^{-4}$ & $4.186427 \times 10^{-8}$ & 4.000002 \\
\hline
\end{tabular}

Tabela 4.3: Resultados numéricos obtidos pelo Método de Crank-Nicolson na aproximação da solução do problema (4.6).

Ao refinar a malha o erro cometido na aproximação do problema (4.6) tende a zero e as razões entre os erros tendem a 4, assim conlui-se que o Método de Crank-Nicolson é de segunda ordem.

\subsubsection{Técnica do Super-Passo}

Fixou-se o parâmetro do Super-Passo em $\nu=0.001$ e a quantidade de subpassos em $N=9$, note que o super-passo de integração no tempo $\Delta T$ obtido pela técnica do Super-Passo não é necessariamente um divisor do limite superior do intervalo de tempo, neste caso $T=\frac{1}{4}$, ou seja, resta um pequeno intervalo entre o instante da última aproximação realizada pela técnica e o instante final.

Para que exista uma aproximação numérica para a solução do problema proposto em (4.6) no instante final, aplica-se o Método de Euler Explícito neste intervalo de tempo restante. Assim os resultados obtidos por refinamento de malha foram. 


\begin{tabular}{|c|c|c|c|c|}
\hline$N_{x}$ & $\Delta T$ & $N_{T}$ & $\|e\|_{\infty}$ & Razão \\
\hline \hline 25 & $2.171086 \times 10^{-3}$ & 1046 & $3.643430 \times 10^{-5}$ & \\
\hline 50 & $5.427716 \times 10^{-4}$ & 4184 & $9.151810 \times 10^{-6}$ & 3.981103 \\
\hline 100 & $1.356929 \times 10^{-4}$ & 16607 & $2.366254 \times 10^{-6}$ & 3.867636 \\
\hline 200 & $3.392323 \times 10^{-5}$ & 66364 & $5.941124 \times 10^{-7}$ & 3.982838 \\
\hline 400 & $8.480807 \times 10^{-6}$ & 265327 & $1.488345 \times 10^{-7}$ & 3.991765 \\
\hline
\end{tabular}

Tabela 4.4: Resultados numéricos obtidos pela Técnica do Super-Passo na aproximação da solução do problema (4.6).

A tabela acima ilustra que quanto maior forem as divisões na direção $x$, menor será o erro cometido na aproximação da solução do problema (4.6). O super-passo de integração no tempo da Técnica do Super-Passo $\Delta T$ depende do passo de integração no tempo do Método de Euler Explícito (esquema (3.14)), mais uma vez escolhido $\Delta t_{\text {expl }}=\frac{\Delta x^{2}}{3 \mu_{\max }}$, onde $\mu_{\max }=\mu(1)=1.5$, assim como os resultados apresentados na $5^{a}$ coluna da tabela (4.4) tendem a 4 quando o passo de integração no tempo $\Delta T$ tende a zero: a Técnica do Super-Passo aplicada am Método de Euler Explícito é de primeira ordem. 


\subsection{Comparação de Performance}

Considere mais uma vez o problema modelo (4.6) com condutibilidade variável, reinserido aqui por conveniência,

$$
\left\{\begin{array}{l}
u_{t}-\left[\mu(x) u_{x}\right]_{x}=f(x, t) \\
u(x, 0)=\cos (\pi x), \quad 0 \leq x \leq \frac{1}{4} ; \quad 0 \leq t \leq \frac{1}{4} \\
u(0, t)=\cos (\pi t), \quad u\left(\frac{1}{4}, t\right)=\frac{\sqrt{2}}{2} \cos (\pi t) \\
\mu_{x}(x)=\frac{1}{2}, \quad \mu(x)=\frac{x}{2}+1
\end{array}\right.
$$

onde $f(x, t)=u_{t}-\left[\mu(x) u_{x}\right]_{x}$ e $u(x, t)=\cos (\pi x) \cos (\pi t)$.

Para as aproximações obtidas consideraram-se 100 divisões no intervalo $0 \leq x \leq \frac{1}{4}$, ou seja, $N_{x}=100$. O tamanho do passo no tempo foi fixado como sendo $\Delta t_{\text {expl }}=\frac{\Delta x^{2}}{3 \mu_{\max }}$, onde $\mu_{\max }=\mu(1)=1.5$. Os métodos implícitos utilizaram passos no tempo dados por $\Delta t=$ cte $* \Delta t_{\text {expl }}$, com cte $=20,40,80,120,200$.

Na tabela abaixo, $N_{T}$ é a quantidade de passos no tempo, $N$ e $\nu$ são parâmetros do super-passo $\Delta T$, o símbolo $\|e\|_{\infty}$ representa o erro máximo cometido no instante final $T=\frac{1}{4}$ e $V_{\text {relativo }}$ quantas vezes o tempo de processamento necessário para se obter a aproximação no instante final de tempo foi mais rápido do que o tempo de processamento gasto pelo Método de Euler Explícito.

Isto posto, a Tabela (4.5) contém as aproximações numéricas obtidas. 


\begin{tabular}{|c|c|c|c|c|c|}
\hline \multicolumn{6}{|c|}{ Super-Passo } \\
\hline$\overline{\Delta T}$ & $N_{T}$ & 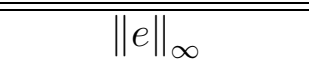 & $V_{\text {relativo }}$ & $\bar{N}$ & $\nu$ \\
\hline $8.281701 \times 10^{-5}$ & 21158 & $1.568263 \times 10^{-6}$ & 6.78 & 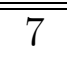 & 0.0015 \\
\hline $1.356929 \times 10^{-4}$ & 16607 & $2.366254 \times 10^{-6}$ & 8.71 & 9 & 0.0010 \\
\hline $1.007775 \times 10^{-4}$ & 22359 & $1.619285 \times 10^{-6}$ & 6.18 & 9 & 0.0050 \\
\hline $2.381135 \times 10^{-4}$ & 21099 & $2.045039 \times 10^{-6}$ & 6.84 & 20 & 0.0060 \\
\hline \multicolumn{6}{|c|}{ Euler Explícito } \\
\hline$\overline{\Delta \Delta t_{\text {expl }}}$ & $N_{T}$ & 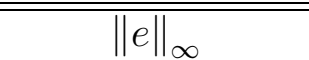 & $V_{\text {relativo }}$ & & \\
\hline $1.851852 \times 10^{-6}$ & 135000 & $3.368690 \times 10^{-7}$ & 1 & & \\
\hline \multicolumn{6}{|c|}{$\begin{array}{ll}\text { Euler Implícito } \\
\end{array}$} \\
\hline cte & $N_{T}$ & $\|e\|_{\infty}$ & $V_{\text {relativo }}$ & & \\
\hline 20 & 6750 & $5.939765 \times 10^{-7}$ & 18.74 & & \\
\hline 40 & 3375 & $1.480069 \times 10^{-6}$ & 40.01 & & \\
\hline 80 & 1687 & $3.253415 \times 10^{-6}$ & 82.90 & & \\
\hline 120 & 1125 & $5.025636 \times 10^{-6}$ & 117.35 & & \\
\hline 200 & 675 & $8.572301 \times 10^{-6}$ & 200.22 & & \\
\hline \multicolumn{6}{|c|}{ Crank-Nicolson } \\
\hline 20 & 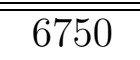 & $2.916720 \times 10^{-7}$ & 21.34 & & \\
\hline 40 & 3375 & $2.929208 \times 10^{-7}$ & 43.00 & & \\
\hline 80 & 1687 & $2.939802 \times 10^{-7}$ & 85.90 & & \\
\hline 120 & 1125 & $2.955703 \times 10^{-7}$ & 127.35 & & \\
\hline 200 & 675 & $3.008692 \times 10^{-7}$ & 209.72 & & \\
\hline
\end{tabular}

Tabela 4.5: Erros cometidos nas aproximações da solução da equação (4.7).

Os resultados numéricos obtidos pela Técnica do Super-Passo para aproximar a solução do problema (4.7) tiveram um tempo de processamento inferior aos obtidos pelo Método de Euler Explícito. A aproximação obtida escolhendo-se $N=9$ e $\nu=0.001$ foi quase 9 vezes mais rápida que a obtida pelo Método de Euler Explícito, A Técnica do Super-Passo obteve uma aproximação mais precissa com os parâmetros $N=7$ e $\nu=0.0015$ e teve tempo de processamento aproximadamente 7 vezes mais rápido que o Método de Euler Explícito. 
As aproximações ilustradas na Tabela 4.5 mostram que o Método de Euler Explícito possui um erro menor em relação aos demais métodos. Entretanto, possui um tempo de processamneto pelo menos 6 vezes maior que os demais.

A Técnica do Super-Passo com os parâmetros $N=9$ e $\nu=0.001$ obteve o seguinte erro na aproximação da solução da equação (4.7) $2.136625 \times 10^{-6}$ e o tempo de processamento foi 9 vezes menor que o do Método de Euler Explícito

A aproximação mais precissa obtida pelo Método de Euler Implícito foi realizada escolhendo cte $=20$ e seu tempo de processamento foi aproximadamete 19 vezes mais rápido do que o do Método de Euler Explícito obtendo o seguinte erro na aproximação $5.939765 \times 10^{-7}$. O menor erro cometido na aproximação da solução do problema (4.7) pela Técnica do Super-Passo foi $1.568263 \times 10^{-6}$, com os parâmetros $N=7$ e $\nu=0.0015$ e foi 7 vezes mais rápido que os resultados pelo Método de Euler Explícito, assim a Técnica do Super-Passo e o Método de Euler Implícito tiveram tempos de processamento com a mesma ordem de grandeza.

Pelos valores da Tabela 4.5 percebe-se que os resultados obtidos pelo Método de Euler Implícito e pela Técnica do Super-Passo para o problema modelo (4.7) são comparáveis, mas o mesmo não ocorre com o Método de Crank-Nicolson que realizou aproximações 100 vezes mais precissas, fato já esperado pois se trata de um método de $2^{a}$ ordem, enquanto os Métodos de Euler Explícito e Implícito e Super-Passo são de $1^{a}$ ordem.

A Técnica do Super-Passo possui implementação muito mais simples do que o Método de Euler Implícito, bastam poucas alterações no código do método explícito para se obter a implementação da técnica e obter resultados semelhantes aos obtidos pelo Método de Euler Implícito, como pode ser visto na tabela (4.6).

De modo a ilustrar a simplicidade da implementação da Técnica do Super-Passo partindo de uma implementação já existente do Método de Eu- 
ler Explícito, foi montada a tabela (4.6) que compara os pseudo-códigos do Método de Euler Explícito e Técnica do Super-Passo.

Na tabela abaixo $N$ é a quantidade de sub-passos, $\tau_{i}$ os sub-passos de integração no tempo calculados utilizando a fórmula (3.13), $N_{S T S}$ é quantidade de super-passos no tempo da Técnica do Super-Passo, $N_{T}$ a quantidade de passos no tempo do Método de Euler Explícito, $\Delta t$ e $\Delta t_{\text {expl }}$ passos de integração no tempo, e por último, $N_{x}+1$ a quantidade de pontos no intervalo da variável $x$.

\begin{tabular}{|l|c|c|}
\hline & Euler Explícito & Super-Passo \\
\hline \hline 1 & & do $i=1, N$ \\
\hline & & $\arg =2(i-1) / N$ \\
\hline & & $\tau_{i}=\Delta t_{\text {expl }} /[(\nu-1) \cos (0.5 a r g) \nu+1]$ \\
\hline 4 & & end do \\
\hline & & do $k=1, N_{S T S}$ \\
\hline 6 & do $k=1, N_{T}$ & do $j=1, N$ \\
\hline 7 & & do $i=1, N_{x}-1$ \\
\hline & do $i=1, N_{x}-1$ & Método de Euler com $\Delta t=\tau_{j}$ \\
\hline 9 & Método de Euler com $\Delta t=\Delta t_{\text {expl }}$ & end do \\
\hline & end do & atualizações \\
\hline & atualizações & condições de contorno \\
\hline 13 & condições de contorno & end do \\
\hline & end do & end do \\
\hline
\end{tabular}

Tabela 4.6: Comparação entre os pseudo-códigos da Técnica do Super-Passo e do Método de Euler.

A Tabela (4.6) mostra que para implementar a Técnica do Super-Passo apartir de uma implemantação já existente da Método de Euler Explícito, basta adicionar o loop das linhas 1 à 4 , a troca de $N_{T}$ por $N_{S T S}$ na linha 6 , acrescentar o loop dos sub-passos da Técnica do Super-Passo, linhas 7 e 13, alterar o passo de integração no tempo de $\Delta t_{\text {expl }}$ para $\tau_{j}$, linha 9. 


\section{Capítulo 5}

\section{Conclusão}

O presente trabalho descreve a aplicação e a implementação da Técnica do Super-Passo no caso do Método de Euler Explícito. Tal técnica aumenta a eficiência computacional do Método de Euler Explícito, relaxando a condição de estabilidade imposta ao passo de integração no tempo reduzindo, portanto, o número de cálculos efetuados.

Os resultados obtidos para um problema modelo com solução conhecida sugerem que a Técnica do Super-Passo é uma forma de "reciclar" códigos que fazem uso do Método de Euler Explícito tornando-os mais rápidos e com erros que tem a mesma ordem de grandeza daqueles gerados pelo Método de Euler Implícito sem, entretanto, a necessidade de se resolver qualquer tipo de sistema de equações a cada passo de integração no tempo.

Observe que o caso do problema ser não-linear, faz-se necessária a resolução de um sistema não-linear a cada passo no tempo, o que pode tornar os métodos implícitos muito caros em termos de processamento, e será necessário implementar um método iterativo para aproximar a solução desse sistema não linear.

No Capítulo 3 e pela Tabela (4.6), percebe-se o quão simples é a implementação da Técnica do Super-Passo partindo da já existente implementação 
do Método de Euler Explícito.

Como continuidade do trabalho pode-se desenvolver a teoria do SuperPasso para a aplicação da técnica a métodos explícitos de ordem mais alta. Tal formulação ainda não foi desenvolvida. 


\section{Apêndice}

\section{Polinômios de Chebyshev}

As formulações, e propriedades dos Polinômios de Chebyshev apresentadas neste Apêndice são encontradas em [3], [12],[14],[19], [24].

As funções trigonométricas foram amplamente estudadas durante os anos, e possuem aplicações desde a modelagem matemática de problemas físicos até a representação de números complexos. Existem várias propriedades que envolvem funções trigonométricas, em especial, o fato que $\cos (n \theta), n \geq 0$, pode ser expresso como um polinômio de grau $n$ em $\cos (\theta)$, com $0<\theta<2 \pi$. Os polinômios de Chebyshev são oriundos dessa representação de $\cos (n \theta)$. Alguns exemplos desses polinômios são:

$$
\begin{aligned}
& \cos (2 \theta)=2 \cos ^{2}(\theta)-1, \\
& \cos (3 \theta)=4 \cos ^{3}(\theta)-3 \cos (\theta), \\
& \cos (4 \theta)=8 \cos ^{4}(\theta)-8 \cos ^{2}(\theta)+1, \quad \operatorname{com} \quad 0 \leq \theta \leq 2 \pi .
\end{aligned}
$$

A forma polinômial de $\cos (n \theta)$ em $\cos (\theta)$ de grau $n$ é denotada por 
$T_{n}(\cos (\theta))$. Fazendo a mudança de variáveis $x=\cos (\theta)$ tem-se,

$$
\begin{aligned}
& T_{0}(x)=1, \\
& T_{1}(x)=x \\
& T_{2}(x)=2 x^{2}-1 \\
& T_{3}(x)=4 x^{3}-3 x \\
& T_{4}(x)=8 x^{4}-8 x^{2}+1, \quad-1 \leq x \leq 1 .
\end{aligned}
$$

Pelas expressões acima para $T_{n}(x)$ pode-se escrever a seguinte formula de recursão para os Polinômios de Chebyshev.

Dados $T_{0}(x)=1$ e $T_{1}(x)=x$, com $-1 \leq x \leq 1$, a identidade a seguir é válida

$$
T_{n+1}(x)=2 x T_{n}(x)-T_{n-1}(x), \quad n=2,3, \ldots
$$

A formulação em (5.1) fornece a seguinte propriedade dos polonômios de Chebyshev,

$$
\left|T_{n}(x)\right|>1, \quad \text { para }|x|>1
$$

A relação $T_{n}(\cos (\theta))=\cos (n \theta)$ atribui as seguintes propriedades trigonométricas aos Polinômios de Chebyshev.

- $\left|T_{n}(x)\right| \leq 1$, para $|x| \leq 1$.

- $T_{n}(x)$ possui valores extremos \pm 1 em $x=x_{j}=\cos \left(\frac{\pi}{n} j\right), \operatorname{com} j=$ $0,1, \ldots, n+1$.

- $T_{n}(x)$ possui $n$ raízes distintas em $x=x_{j}=\cos \left(\frac{(2 j+1)}{n} \frac{\pi}{2}\right)$, com $j=0,1, \ldots, n-1$.

Aplicando a mudança de variáveis $x=\cos (\theta)$ em $T_{n}(\cos (\theta))=\cos (n \theta)$ tem-se a forma trigonométrica dos Polinômios de Chebyshev. 


$$
T_{n}(x)=\cos (n \arccos (x)), \quad \text { para } \quad-1 \leq x \leq 1 .
$$

Fazendo a mudança de variáveis $x=\cos (\theta)$ em (5.2) tem-se,

$$
T_{n}(\cos (\theta))=\cos (n \theta)=\frac{1}{2}\{\cos (n \theta)+i \sin (n \theta)+\cos (n \theta)-i \sin (n \theta)\} .
$$

Pelo teorema de Moivre obtem-se,

$$
\begin{aligned}
T_{n}(\cos (\theta)) & =\cos (n \theta)=\frac{1}{2}\{\cos (n \theta)+i \sin (n \theta)+\cos (n \theta)-i \sin (n \theta)\} \\
& =\frac{1}{2}\left\{(\cos (\theta)+i \sin (\theta))^{n}+(\cos (\theta)-i \sin (\theta))^{n}\right\}
\end{aligned}
$$

Lembre que $i=\sqrt{-1}$ e $\cos ^{2}(\theta)-1=-\sin ^{2}(\theta)$, assim,

$$
\begin{aligned}
T_{n}(\cos (\theta)) & =\frac{1}{2}\left\{(\cos (\theta)+i \sin (\theta))^{n}+(\cos (\theta)-i \sin (\theta))^{n}\right\}, \\
& =\frac{1}{2}\left\{\left(\cos (\theta)+\sqrt{\cos ^{2}(\theta)-1}\right)^{n}+\left(\cos (\theta)-\sqrt{\cos ^{2}(\theta)-1}\right)^{n}\right\} .
\end{aligned}
$$

Voltando a variável original $x$ tem-se a seguinte expressão para o Polinômio de Chebyshev.

$$
T_{n}(x)=\frac{1}{2}\left\{\left(x+\sqrt{x^{2}-1}\right)^{n}+\left(x-\sqrt{x^{2}-1}\right)^{n}\right\}
$$

onde $-1 \leq x \leq 1$.

Da formulação acima segue que o polinômio de Chebyshev é par se $n$ for par e ímpar se $n$ for impar, de fato. 
Se $n=2 m, m \in Z_{+}^{*}$,

$$
\begin{aligned}
T_{2 m}(-x) & =\frac{1}{2}\left\{\left(-x+\sqrt{x^{2}-1}\right)^{2 m}+\left(-x-\sqrt{x^{2}-1}\right)^{2 m}\right\}, \\
& =\frac{1}{2}\left\{\left(-1\left(x-\sqrt{x^{2}-1}\right)\right)^{2 m}+\left(-1\left(x+\sqrt{x^{2}-1}\right)\right)^{2 m}\right\}, \\
& =\frac{1}{2}\left\{\left(x+\sqrt{x^{2}-1}\right)^{2 m}+\left(x-\sqrt{x^{2}-1}\right)^{2 m}\right\}=T_{2 m}(x) .
\end{aligned}
$$

Logo $T_{n}(x)$ é uma função par se $n$ for par.

Se $n=2 m+1, m \in Z_{+}$,

$$
\begin{aligned}
T_{2 m+1}(-x) & =\frac{1}{2}\left\{\left(-x+\sqrt{x^{2}-1}\right)^{2 m+1}+\left(-x-\sqrt{x^{2}-1}\right)^{2 m+1}\right\}, \\
& =\frac{1}{2}\left\{\left(-1\left(x-\sqrt{x^{2}-1}\right)\right)^{2 m+1}+\left(-1\left(x+\sqrt{x^{2}-1}\right)\right)^{2 m+1}\right\}, \\
& =\frac{1}{2}\left\{-\left(x-\sqrt{x^{2}-1}\right)^{2 m+1}-\left(x+\sqrt{x^{2}-1}\right)^{2 m+1}\right\}, \\
& =-\frac{1}{2}\left\{\left(x-\sqrt{x^{2}-1}\right)^{2 m+1}\left(x+\sqrt{x^{2}-1}\right)^{2 m+1}\right\}=-T_{2 m}(x) .
\end{aligned}
$$

Logo $T_{n}(x)$ é uma função ímpar se $n$ for ímpar. 
A Figura abaixo ilustra o gráfico de alguns Polinômios de Chebyshev.

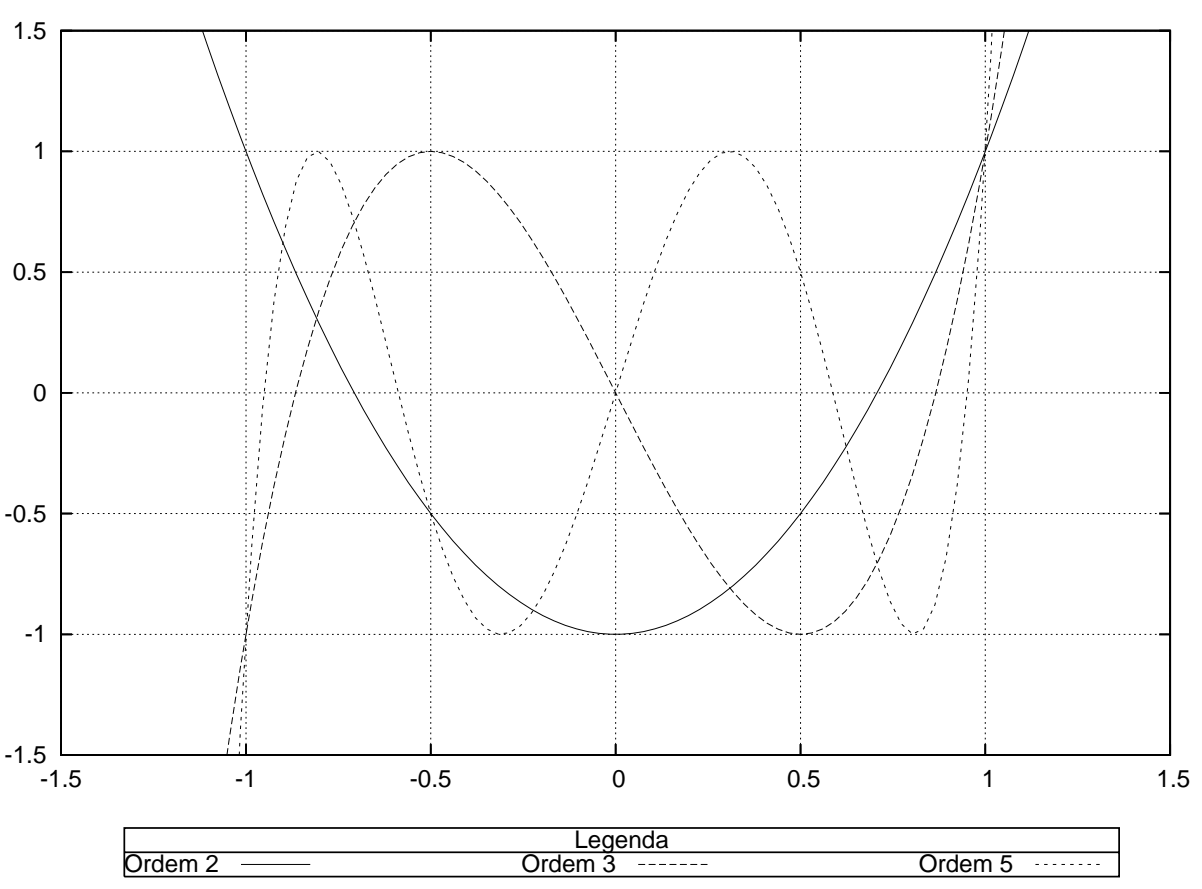

Figura 5.1: Polinômios de Chebyshev.

A Figura (5.1) permite visualizar os Polinômios de Chebyshev de segundo, terceiro e quinto grau, e as propriedades $\left|T_{N}(x)\right| \leq 1$ para $|x| \leq 1$ e que $\left|T_{N}(x)\right|>1$ para $|x|>1$.

Alguns problemas são resolvidos em um intervalo genérico $[a, b]$ e não necessariamente no domínio $[-1,1]$ onde os polinômios de Chebyshev são definidos. Nestes casos se faz necessário realizar uma mudança de variáveis, para que o problema seja resolvido no domínio $[-1,1]$, essa mudança é dada por:

$$
x=\frac{b-a}{2} t+\frac{a+b}{2}
$$

com $a \leq x \leq b$ e $-1 \leq t \leq 1$.

Assim as raízes do polinômio de Chebyshev para um intervalo genérico 
$[a, b]$ são dadas por:

$$
x_{j}=\frac{b-a}{2} \cos \left(\frac{(2 j+1) \pi}{2 n}\right)+\frac{a+b}{2},
$$

para $j=0,1, \ldots, n-1$. 


\section{Referências Bibliográficas}

[1] V. Alexiades. Overcoming the Stability Restriction of Explicit Schemes via Super-Time-Stepping. 2nd International Conference on Dynamic Systems and Applications, Atlanta. 1995.

[2] V. Alexiades, G. Amiez, P. Gremaud. Super-time-stepping acceleration of explicit schemes for parabolic problems. Communications in Numerical Methods in Engineering, 1996.

[3] K. E. Atkinson. Elementary numerical analysis. New York, John Wiley, 425 p., 1993.

[4] R. L. Burden, J. D. Faires. Análise Numérica. Pioneira Thomson Learning, 1st ed., 2003.

[5] B. Carnahan, H. A. Luther, J. O. Wilkes. Applied numerical methods. New York, John Wiley, 604 p., 1969.

[6] S. J. Chapman. Introducting to Fortran 90/95. Springer-Verlag, WCB/McGraw-Hill, 4nd ed., 1998.

[7] R. Courant. Differential and integral calculus. London, Blackie, 2nd ed. 1953.

[8] J. A. Cuminato, M. Meneguette, Jr. Discretização de equações diferenciais parciais - Técnicas de diferenças finitas. 1st ed., 2000. 
[9] B. Epstein. Partial Differential Equations: An Introduction. USA, McGraw-Hill Book Company, 1962.

[10] S. Evje, K. Hvistendahl Karlsen, K. A. Lie, N. H. Risebro. Front Tracking and Operator Splitting for Nonlinear Degenerate Convection-Diffusion Equations. Parallel Solution of Partial Differential Equations, IMA Volumes in Mathematics and its Applications 120, 2000.

[11] D. G. Figueiredo. Análise de Fourier e Equações Diferenciais Parciais. Rio de Janeiro, IMPA, 274 p., 1997.

[12] L. Fox, I. B. Parker. Chebyshev polynomials in numerical analysis. Universities Press, 1st ed.,1968.

[13] W. Gentzsch, A. Schluter. On One Step Methods With Cyclic Stepsize Changes for Solving Parabolic Differential Equationes. 1978.

[14] C. F. Gerald, P. O. Wheatley. Applied Numerical Analysis. AddisonWesley Pub, 1994 .

[15] P. M. Gustavo. Introdução às equações diferenciais parciais. Rio de Janeiro, IMPA, 252 p., 1977.

[16] R. Iório, Jr. , V. M. Iório Equações Diferenciais Parciais: Uma Introdução. Rio de Janeiro, CNPq, 372 p., 1988.

[17] F. John. Partial Differential Equations. Springer-Verlag, 4nd ed.,1991.

[18] R. W. Lewis, I. Masters, J. T. Cross. Automatic timestep selection for the Super-time-stepping acceleration on unstructured grids using objectoriented programming. Communications in Numerical Methods in Engineering, vol. 13, 1997. 
[19] J. H. Mathews. Numerical methods for mathematics, science, and engineering. Prentice Hall, 1st ed., 1992.

[20] H. F. Nordhaug. On Solving a Three Phase Flow Model With Cappillary Forces., 88 p., 2002.

[21] S. O'Sullivan, T. P. Downes. An Explicit Scheme for Multifluid Magnetohydrodynamics. , 2005.

[22] H. Rutishauser. Lectures on Numerical Mathematics. Boston, Birkhäuser, 546 p., 1990.

[23] F. Scheid. Analise numérica. Lisboa, McGraw-Hill, 2nd ed., 617 p., 1991.

[24] M. A. Snyder. Chebyshev methods in numerical approximation. PrenticeHall Inc., 2nd ed.,1966.

[25] J. C. Strikwerda. Finite difference schemes and partial differential equations. Chapman and Hall, 1989.

[26] P. J. Van der Houwen. Construction of Integration Formulas for Initial Value Problems. North-Holland, 1977.

[27] P. J. Van der Houwen. The Development of Runge-Kutta Methods for Partial Differential equations. 14th IMACS World Congress, Atlanta, GA. 1994.

[28] J. G. Verwer, W. H. Hundsdorfer, B. P. Sommeijer. Convergence properties of the Runge-Kutta-Chebyshev method. Centre for Mathematics and Computer Science.

[29] R. Vulanovic. Super-Time-Stepping for Nonlinear Singular Perturbation Problems. Nonlinear Analysis, Theory, Methods and Applications, Vol 30, $\mathrm{N}^{o}$ 1, p. 67-72, 1997. 